\title{
APPLICATION OF RECENT STATIC ADHESION DATA TO THE ADHESION
}

\section{THEORY OF FRICTION}

\section{by}

D.V. Keller, Jr.

Paper presented to 13th Sagamore Conference on

"Physical and Chemical Characteristics of Surfaces and Interfaces" August, 1966

(to be published Syracuse University Press, 1967)
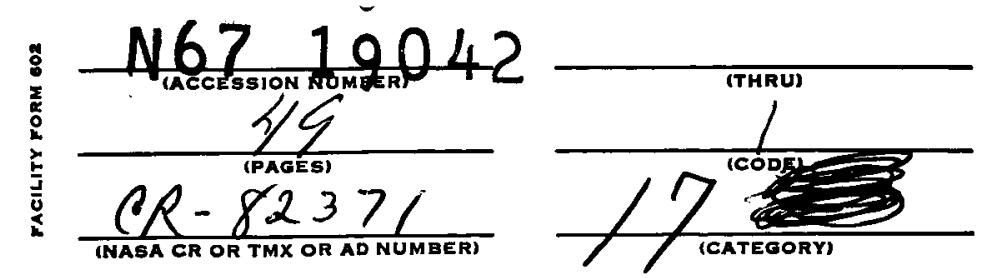
APPLICATION OF RECENT STATIC ADHESION DATA TO THE ADHESION

THEORY OF FRICTION

by

D.V. Keller, Jr.

Syracuse University

The generally accepted theory of friction usually includes an adhesion parameter which in some schools has been contested and in others misinterpreted. The misunderstanding of the position of adhesion in friction relationships is usually based on the lack of knowledge of adhesion phenomena since reliable adhesion data are rather scarse and nearly as complex to interpret as the friction data to which they are applied. An examination of the variables influencing metallic friction as developed from recent vacuum friction experiments will be reviewed briefly and compared to recent observations from static adhesion experiments between clean and specifically contaminated metal surfaces in order to establish a more sound basis far the adhesion theory of friction. 


\section{INTRODUCTION}

The phenomenon incidental to the interaction of two solid surfaces is most commonly observed as friction. Friction, however, by definition alone $(1,2)$ is the mechanical resistance to motion of one surface across another; and, therefore, is not related directly to those fields which promote the creation of an interface, but with those forces which inhibit the separation of such an interface. This point of definition may seem quite small, or even inconsequential; but, as the mechanism of friction is explored in more detail, it will become apparent that the role of interfacial interactions and the ability of the resulting interfaces to be fractured must be maintained separate.

Since friction is the mechanical resistance to motion, the phenomenon bears virtually no material limits and encompasses atomic, molecular, macromolecular or bulk behavior interacting with solids, liquids or gases in nearly every conceivable configuration. The breadth of the subject alone suggests why the literature on the subject is so extensive, yet the agreement on fundamental relationships is so restricted. For example, there is little wonder that the interpretation of the data observed in the study of the frictional behavior of animal joints is most difficult to rationalize by a metallurgist specializing in bearing materials even though the phenomenon involved in both cases is friction. When the study is limited to bulk friction of solids, the true interdisciplinary nature of the subject is unfolded since all structural materials must be included, e.g. metals, alloys, plastics, wood, inorganic chemicals as oxides, nitrides, etc., as well as their respective natural or unnatural lubricants which might include water, oils, silicones, etc. In order to examine a one small facet of this subject in detail, let us restrict our system generally to the kinetic friction of metals in the so-called "dry" condition, that is 
unnatural lubricants have not been added to the system. With such a system a number of the problems required to complete our understanding of the mechanism of friction can be readily emphasized and an approach to an interdisciplinary solution to these problems revealed.

Most recent analysis of the laws of friction $(1,2)$ seem to accept those hypothesis' developed in the 17 th and 18th centuries which suggested that the frictional force acting between two bodies in contact was proportional to the normal force, and, independent of the apparent area of contact. Updating these views the modern concept envisions the frictional force as that force required to shear interfacial mechanical interlocks due to surface roughness as well as "cold-welded" junctions, which occur along the interface of the system. The proportionality constant relating the normal force to the tangential force to maintain motion is called the coefficient of friction $(\mu)$, which may vary from about 0.001 for rolling friction to as high as 5 for galling friction during a sliding process with intermediate values of about 0.2 for simple sliding systems. A distinction has been made by certain authors $(1,2)$ between static and kinetic friction; however, the cause of the former has been suggested to be merely a peculiarity of the measuring system (3) rather than a separate phenomenon. In any event, the static aspects of friction more probably fall under adhesion phenomena, which will be considered later under that title.

Before becoming involved with the interactions which actually promote the formation of the interface in this system, it would be instructive to first examine the mechanical changes which take place at an interface as a spherically-tipped metal rod, or rider, moves relative to flat surface of a second metal. Antler (4) and others (5), have classified the wear processes into four categories as indicated in Figure 1. Wedge formation 

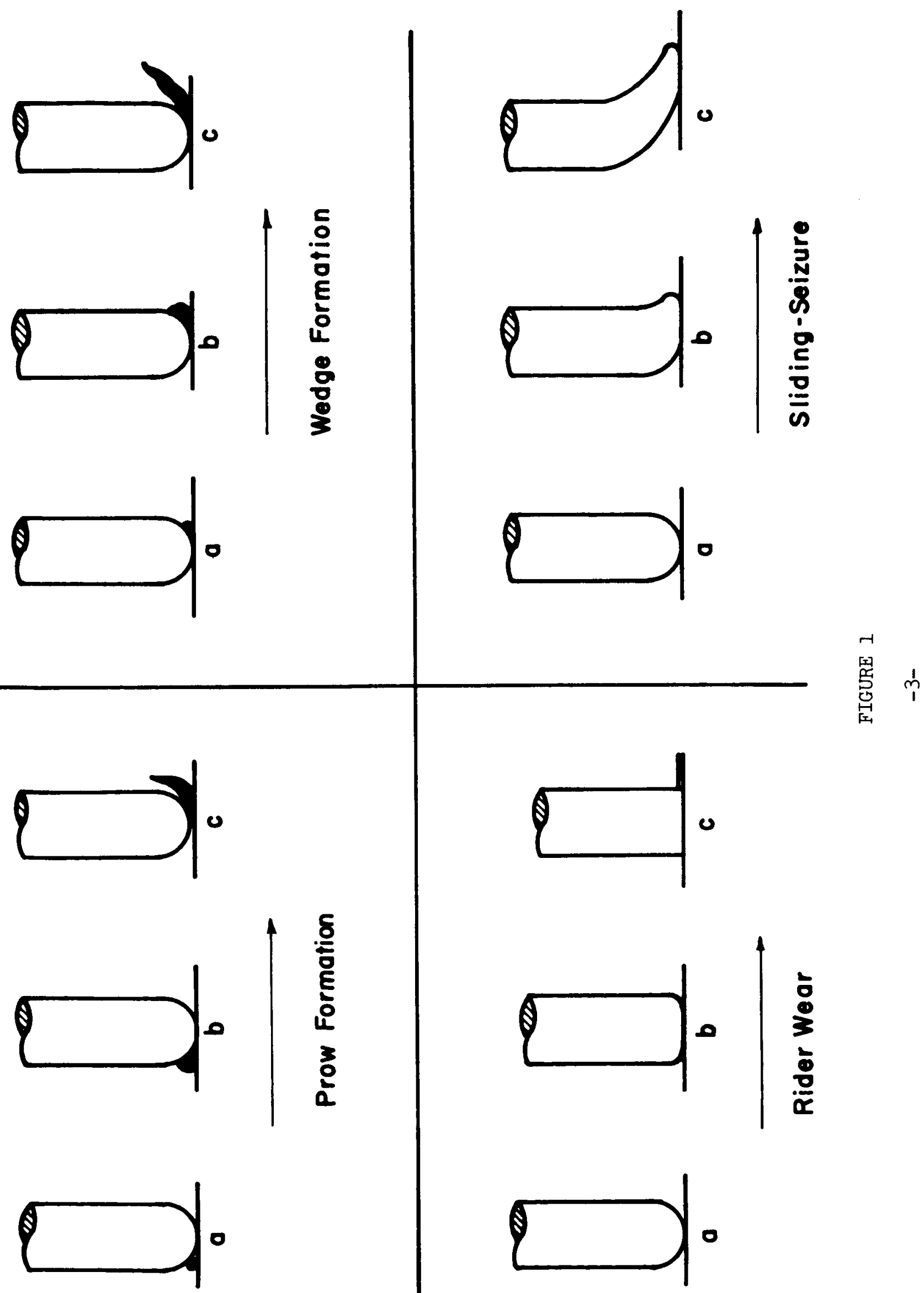
and sliding seizure are characteristic of very soft metals and alloys such as indium and the lead-tin alloys. Prow formation was observed with most face-centered cubic metals until the wear track, formed by the reciprocal motion of the rider on the plate, was fully contaminated with the rider metal; at this time, the rider wear mechanism was observed, e.g. in the case of aluminum sliding on aluminum under heavily loaded conditions, one would expect to observe the rider wear phenomena. This illustration serves to point out the fact that simple friction is intimately related to the deformation and fracture processes continuously occurring in the interface region as the rider progresses along the track; and, as a consequence, a mechanically stable interface must be reformed instantaneously as the indentor is moved along.

The variables in the system are most numerous, e.g. deformation properties of the rider, deformation properties of the plate, interfacial strength between the rider and the plate relative to that of the softer metal, interaction due to surface roughness, etc. Each of these variables, in turn, are also dependent upon the temperature and ambient contaminants at the point of contact. The effects of a number of these variables have been examined in numerous recent reviews on the subject $(1,2)$, to cite a few.

Steijn (6) recently investigated the coefficient of friction between diamond stylus' of varying diameters below $200 \mu$ and various polycrystalline, and single crystal metallic plates. The data was examined utilizing an analysis suggested by Goddard and Wilman (7) in which the total coefficient of friction $\left(\mu_{T}\right)$ on an isotropic surface for shallow indentations was subdivided into that due to adhesion, or the cold-welding, processes $\left(\mu_{a}\right)$, and that due to plowing, or mechanical interlocking phenomena $\left(\mu_{p}\right)$, 


$$
\mu_{T}=\mu_{Q}+\mu_{p}
$$

where the respective values of $\mu_{a}$ and $\mu_{p}$ can be further expanded to

$$
\mu_{T}=k_{a} S L^{(2-n) / n}+k_{p} \rho_{m}^{\prime} L^{(3-n) / n},
$$

where $k_{a}$ and $k_{p}$ are the adhesion and plowing coefficients, $\underline{S}$ is the shear stress of the indentor-stylus interface, $\rho_{m}^{\prime}$ is the mean dynamic flow pressure, $\underline{I}$ is the load on the stylus and $\underline{n}$ is a system constant called the Meyer's index (8). The lower limiting value of $\underline{n}$ is two for an ideally' plastic or a fully work hardened material. On application of the principle of geometric similarity ( 8 ), the effect of indentor diameter (D) may be introduced,

$$
\mu_{T}=k_{a} S L^{(2-n) / n} D^{2(2-n) / n}+K_{p} \rho_{m}^{\prime} L^{(3-n) / n} D^{[2(n-3) / n]}
$$

and the relative contribution of plowing versus adhesion is given by the ratio,

$$
\frac{k_{p}}{k_{a}} \cdot \frac{\rho_{m}^{\prime}}{S} \cdot \frac{L^{I / n}}{D^{2 / n}}
$$

That is, for higher loads and the same indentor diameter, plowing action is favored. Examination of the data from a diamond stylus moved on a polycrystalline copper plate plotted on log-log coordinates serves as an example of this behavior as shown in Figure 2. The coefficient of friction curve for a similar experiment utilizing an indentor of tip radii of $12.7 \mu$ superimposed almost exactly on the $\mu_{p}$ curve suggesting that the adhesion component was almost zero when the tip radii is grossly reduced, i.e. the load drastically increased. From these data, which are supported by a large number of other studies (1), we may conclude that the force of friction is due to that force required to shear the mechanical interlocks 


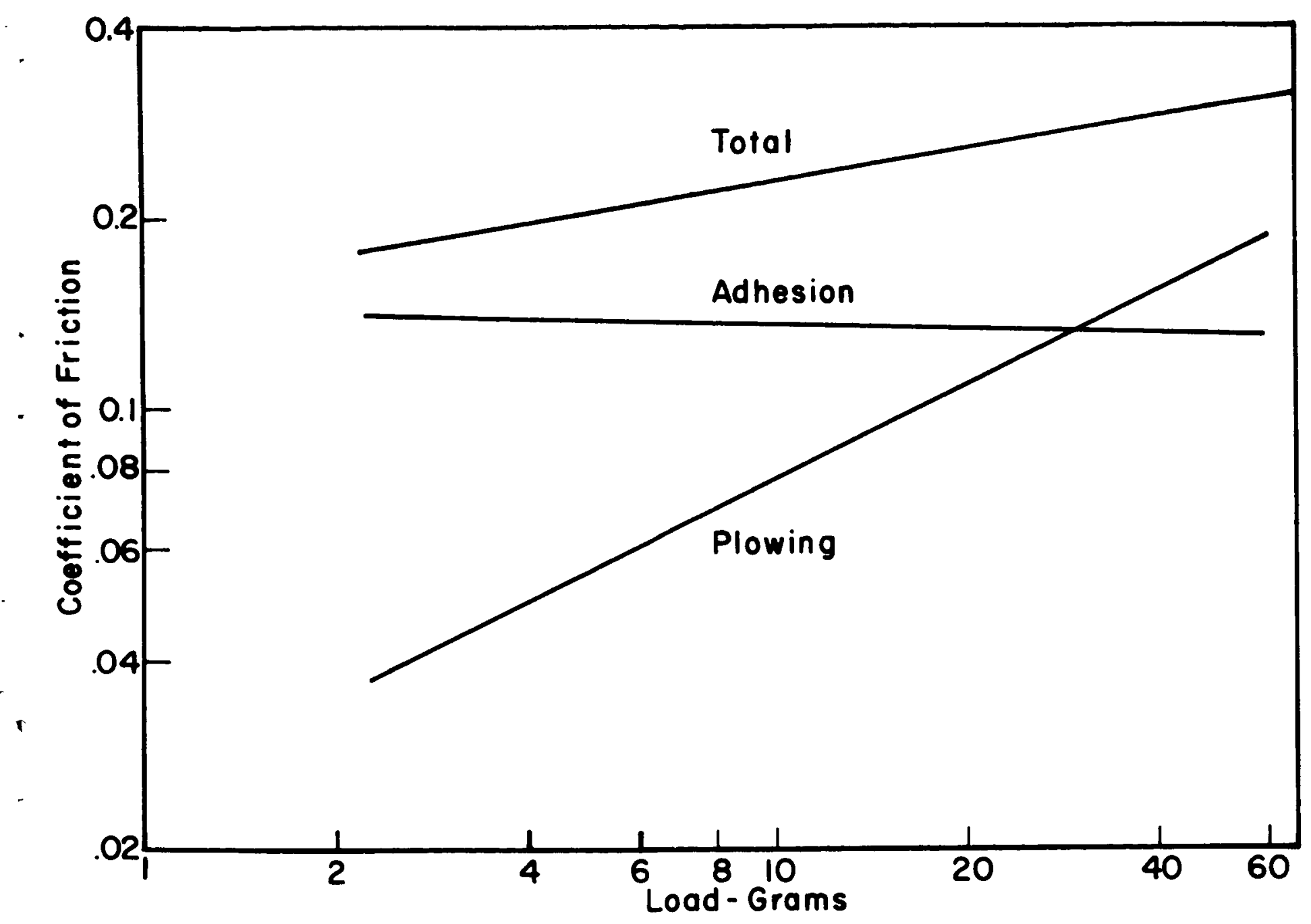

FIGURE 2 - Variation in the plowing and adhesion components of the coefficient of friction and the load on a $76 \mu$ radius diamond stylus moving on polycrystalline copper (8). 
along the interface and the mechanically strong interfaces formed through the process of adhesion. If the surfaces can be considered ideally flat, a case which is rarely observed in practice, the frictional force $(F)$ can be represented by

$$
F=A s
$$

where A represents the true, or real area, of contact and $\underline{s}$ the shear strength of the weaker material (1). The utilization of this approach to adhesion phenomena to friction phenomena, or vise versa, is based on a number of very weak assumptions as will be discussed below.

Utilizing this same technique with an acknowledgement of the effect of anisotropy on the various parameters, Steijn also examined single crystals of aluminum, copper, nickel, $\alpha$ Brass, B Brass, $\alpha$ iron and a number of ionic crystals. Generally, the coefficient of friction on the (100) face varied with direction of motion such that $\left|\mu_{\langle 110>}>\mu_{<100>}\right|$ was observed for all types of crystals.

Since the rider in this case was made of diamond, which is not readily deformable and in a relative manner was heavily loaded, it is not surprising that in most cases the plowing parameter of the friction equation controlled the analysis. This situation could be compared to the tip of a hardness inductor as it penetrates a soft metal, i.e. the tip is not continually meeting a metal oxide surface, but slicing through what may be considered very clean metal surfaces. As a consequence of this, the results are directly related to the deformation properties of the substrate (single crystal) and not grossly effected by the ambient conditions surrounding the surface film. Most friction studies, on the other hand, involve much larger apparent areas of contact, i.e. lighter loads; and most usually, two deformable surfaces such as a metal rider on a metal substrate. Under such conditions, other variables are immediately introduced. As

$$
-7-
$$


suggested by Steijn's paper, the adhesion parameter appears to become important under these conditions, and as suggested by Antler's paper deformation of both surfaces leads to wear phenomena. Since fracture is an intimate part of friction and as we have learned from Westwood's (9) and Bryant's (10) investigations of environmental effects on fracture, one should expect, too, that the same situation applies directly to friction phenomena. The investigations of Roberts and Owens (12) have confirmed this. Entirely neglecting the gross effects of prepared lubricants, let us momentarily examine just the effects of air which normally contains more than an adequate concentration of oxidizing agents to immediately reduce any available free metal atoms exposed on a free surface. Buckley, Schwebert and Johnson (11) have shown, as represented in Figure 3, the variation of the total friction coefficient between a 52100 steel sliding on 52100 steel with the change in ambient atmospheric pressure at a fixed time, which, in turn, reflects on the concentration of available oxidizing agents in the interface system. However, if the majority of gases are eliminated from the system by helium cryogenic pumping, the results illustrated complete welding of a pair of metals which are used normally in bearing systems as shown in Figure 4.

The decrease in $\mu_{\mathrm{T}}$ shown in Figure 3 was accounted for by a change in the wear mechanism in that under atmospheric conditions the wear particles were oxidized fully and removed from the system, while in vacuum at $10^{-6}$ Torr the availability of oxygen was reduced and a rider mechanism was observed. The Iong term studies shown in Figure 4 indicate that in time, the system will form a completely welded junction.

It is evident from these examples that friction studies directed toward the understanding of the mechanism of friction must either totally eliminate, or carefully content with, the presence of natural lubricants 


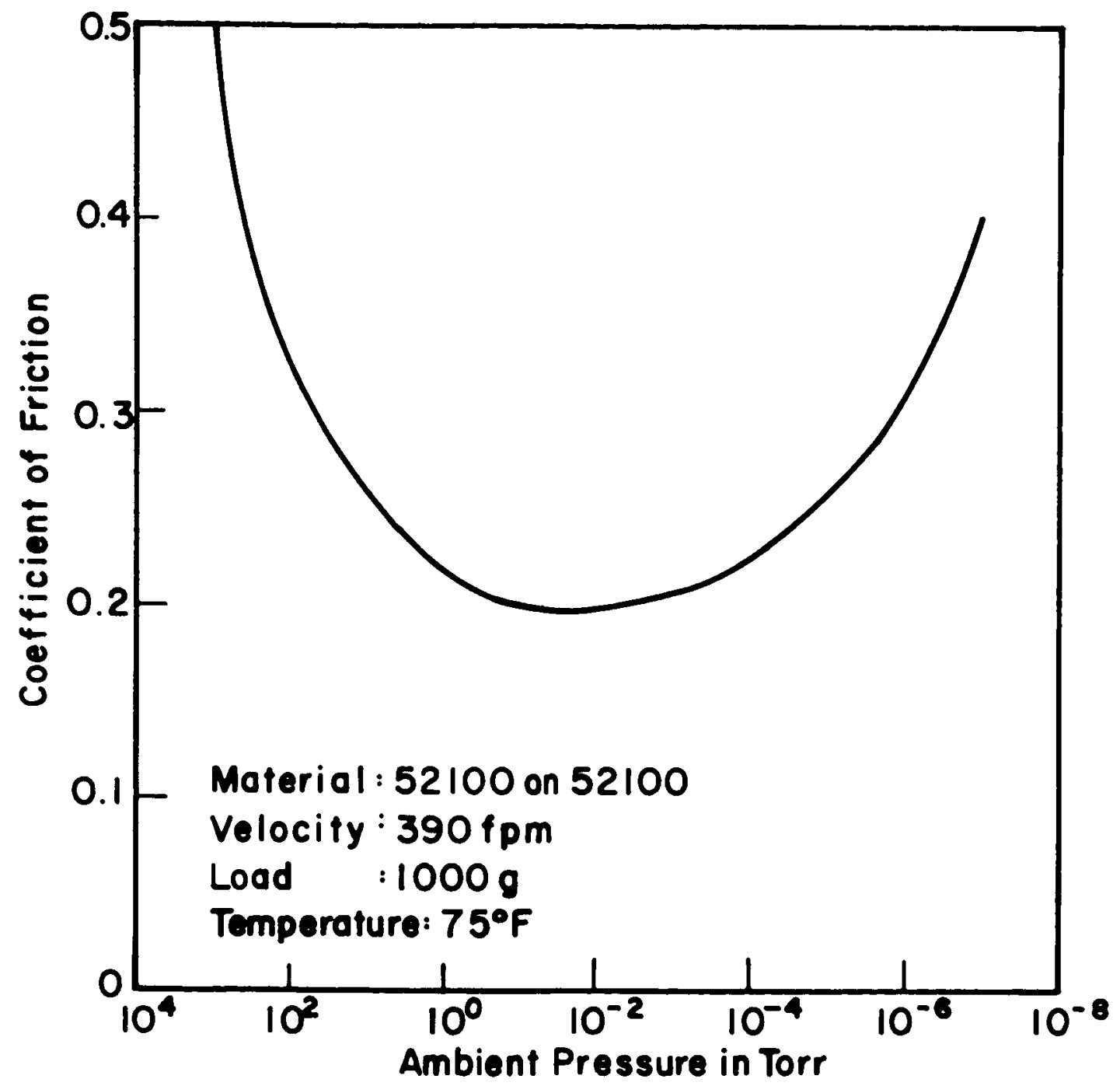

FIGURE 3 - The change in the coefficient of friction with a change in ambient pressure (11).

$-9-$ 


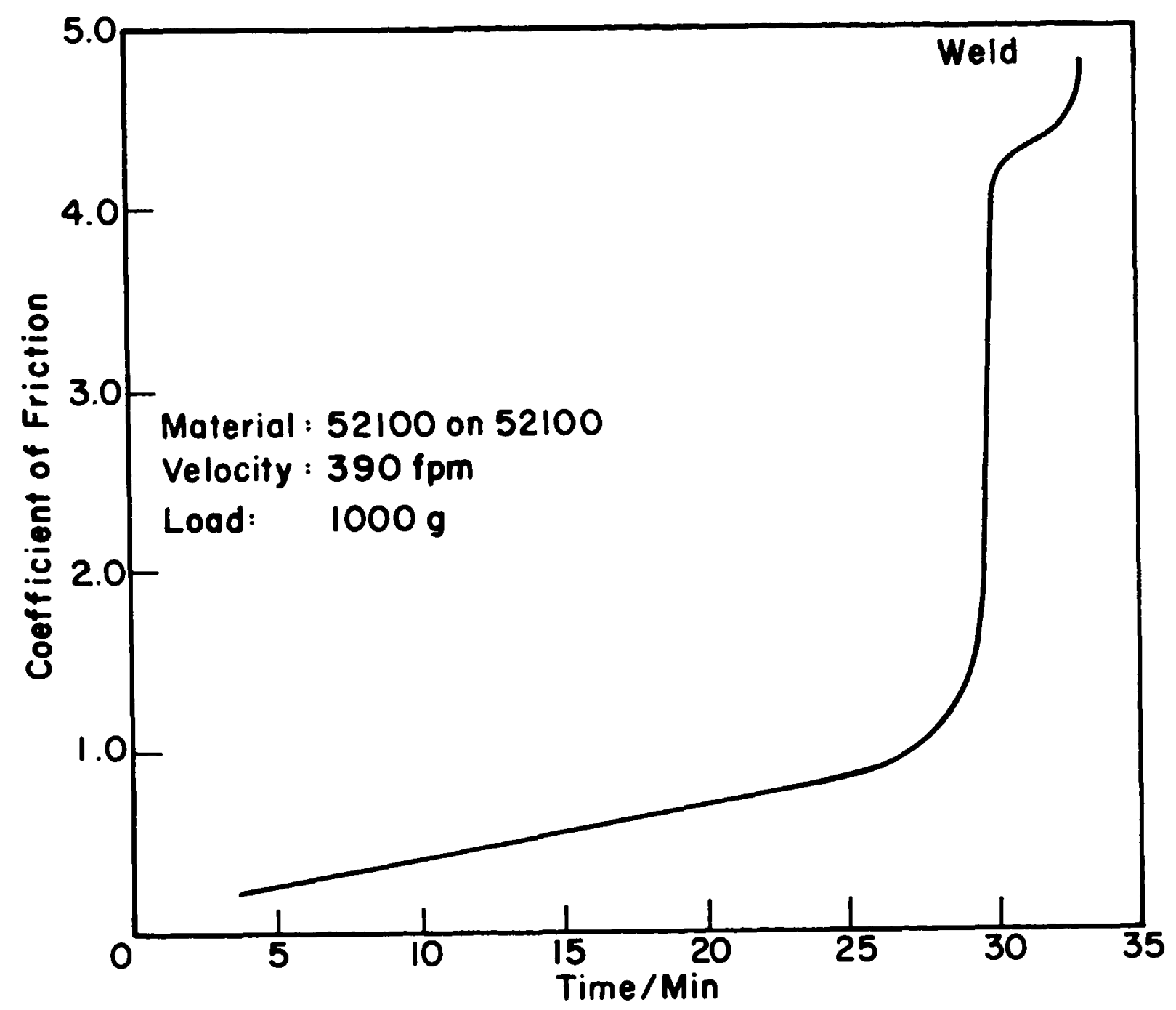

FIGURE 4 - Coefficient of friction for 52100 on 52100 sliding in vacuum with liquid helium cryogenic cooling at ambient pressures in the range of $2 \times 10^{-1}$ Torr (11).

$-10-$ 
such as water vapor, oxygen, nitrogen, etc. The effect of such common commodities as these on simple sliding systems or mildly lubricated systems are rarely considered since their complete elimination from a test system is most difficult as indicated by the numerous surface studies on pure metal surfaces reviewed in this symposium. Before becoming involved with the finer details of contamination, however, let us first examine the literature for other significant variables which effect the coefficient of friction; and in order to observe these more clearly, let us restrict our examination to only high vacuum experiments in which the concentration of the majority of the oxidizing agents can be considered as very low. The majority of data cited in the following few paragraphs were obtained in an ion and/or helium cryogenic pumped vacuum system at pressures below $10^{-9}$ Torr. The $3 / 16 "$ radius stationary rider was loaded on to a rotating flat plate, which moved at variable velocities to $750 \mathrm{~cm} / \mathrm{sec}$ and loads which could be varied to 1500 gms. For the most part, these works were conducted at under the direction of R.L. Johnson (3,13-19). The effect of sliding velocity on the coefficient of friction is shown in Figures 5 and 6 . In Figure 5 the coefficient of friction $(\mu)$ is plotted for a number of metals (rider) sliding on 440-C stainless steel plates. It is evident that the shape of the curve is a function of the slider material of the friction couple; for example, the addition of $21 \mathrm{~A} / \mathrm{O}$ aluminum to titanium completely changes the nature of the titanium curve (14). Pure titanium wears by the rider wear mechanism, whereas the alloy wears by a process similar to that of prow formation, e.g. the track is worn rather than the indentor. Figure 6 supports that which was suspected all along, the faster the rider travels relative to the plate, the more heat that is generated at the interface and at some point melting along the interface will occur. What was not intuitively obvious was the fact that hexagonal metals seem to demonstrate much 


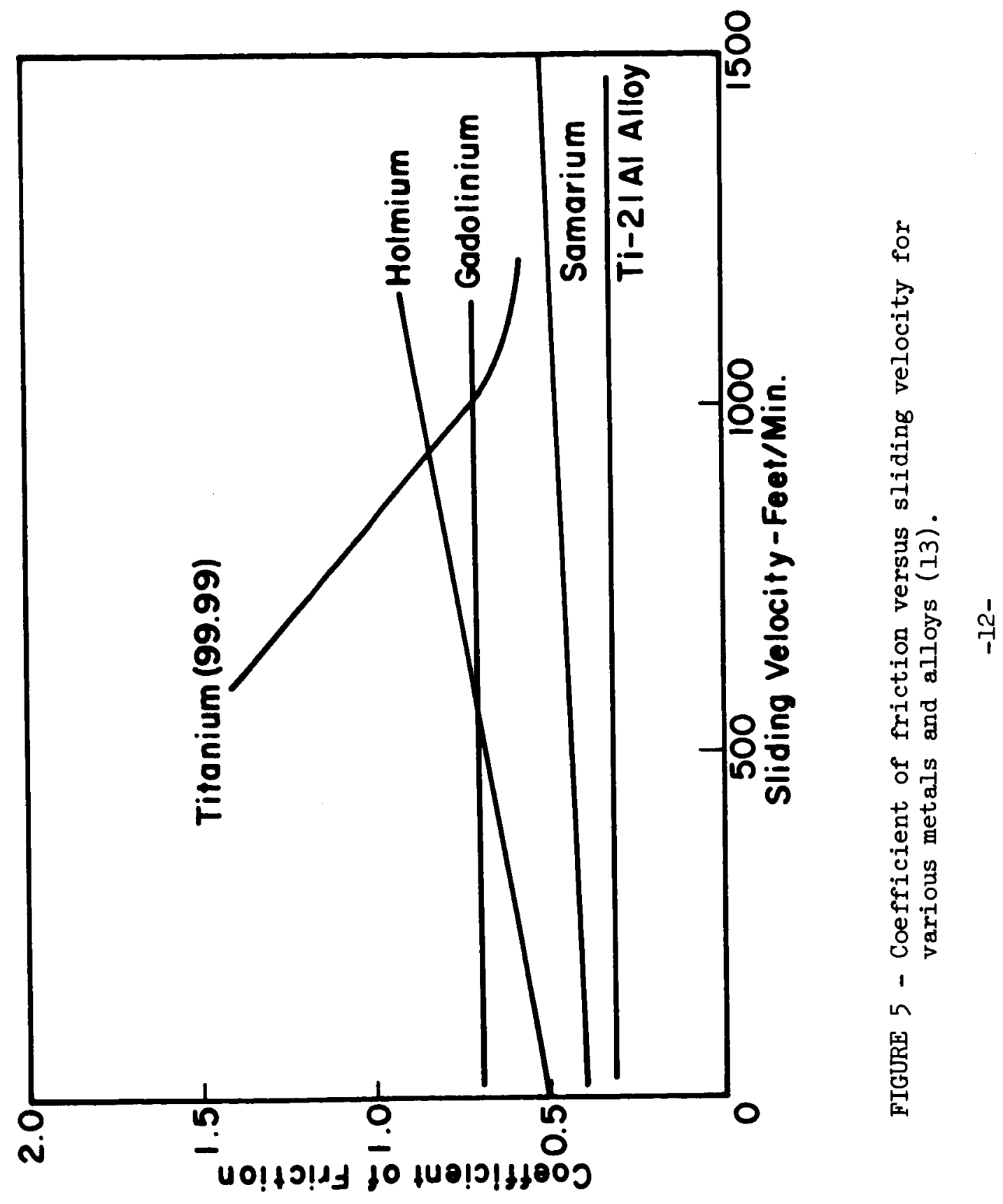




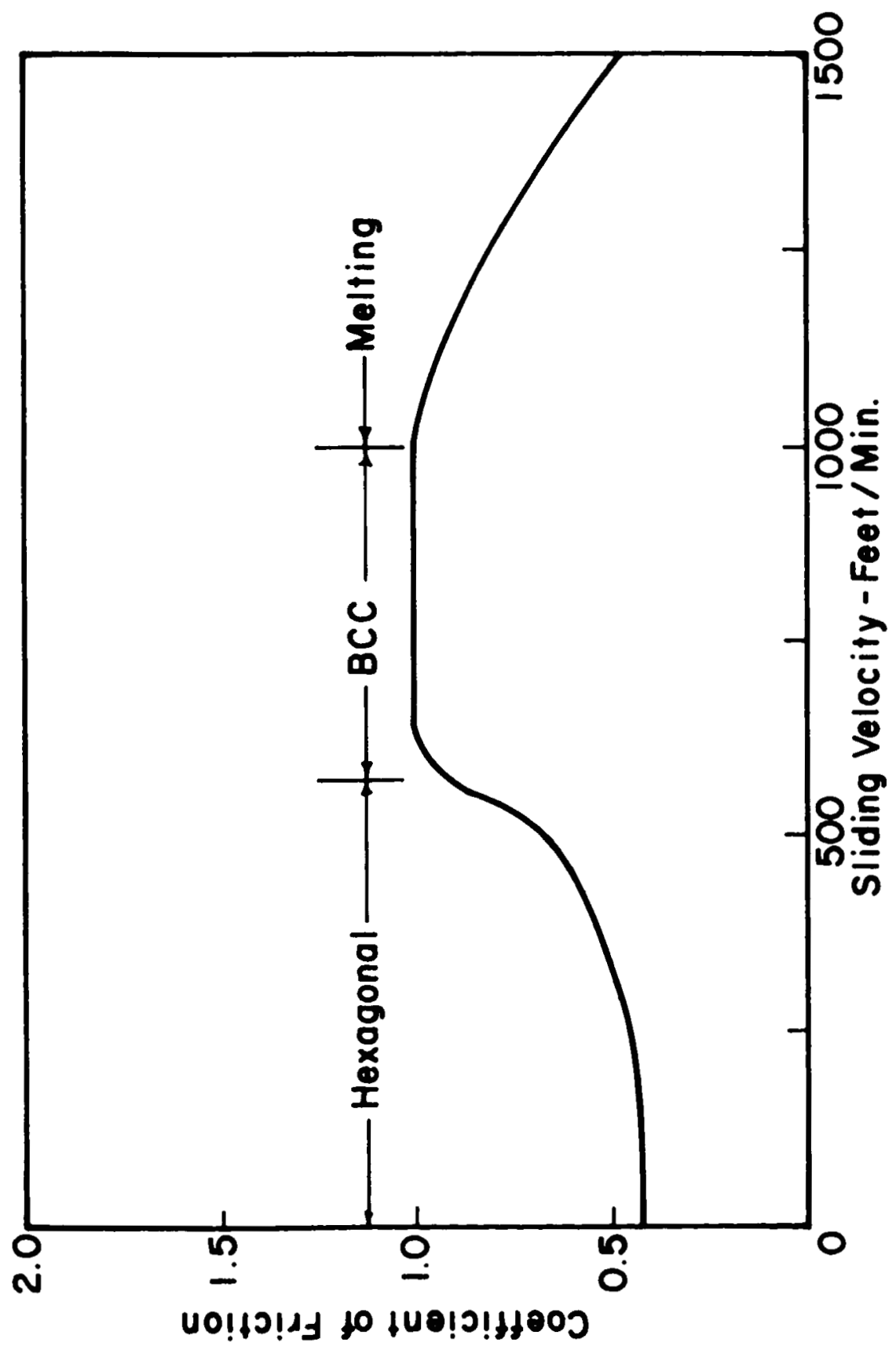

FIGURE 6 - Coefficient of friction versus sliding velocity for thallium on 440-C steel. 
lower frictional coefficients than other structures. This has been confirmed by studying the cobalt-cobalt system (15) as a function of slider velocity as well as temperature, and there is a marked change at the $\alpha-\beta$ transition point to support this hypothesis. Carrying the effect of structure somewhat further, Buckley et al have shown that the effect of structure on $\mu_{\mathrm{T}}$ is real by examining the change in $\mu_{\mathrm{T}}$ with a change in $\frac{c}{a}$ ratio for various hexagonal metals as shown in Figure 7 .

Investigation of single crystal beryllium riders sliding on beryllium (16) further confirms the fact that the critical resolved shear of the material plays an important role, since the basal orientation produced much lower $\mu_{T}$ values than when the crystal was oriented with its prismatic plane parallel to the plate. Similar variations in $\mu_{\mathrm{T}}$ versus crystallographic orientations were observed when a sapphire rider was moved across a number of tungsten single crystals in a large plate (17). Variation in the orientation of the sapphire also seemed to change the orientation friction relationships, which were observed on the tungsten crystals. Grain boundary displacement plays a role in the deformation process.

The few examples cited above should suffice to illustrate that with the elimination of natural lubricants, air, water, etc., the other variables affecting the coefficient of friction can be examined with a fair degree of detail. The role of a third component in the system, the lubricant, whether it is natural or unnatural, serves to prevent the formation of the mechanically strong junctions, e.g. in the case under consideration the formation of metal-metal bonds; and, in turn, supplements the low shear stress interactions of the third component since the surfaces are still in contact and the interface is still in a state of relative motion. Excellent evidence for this is shown in Figure 8 in which the coefficient of friction of a polyimide lubricant was studied as a function of the number 


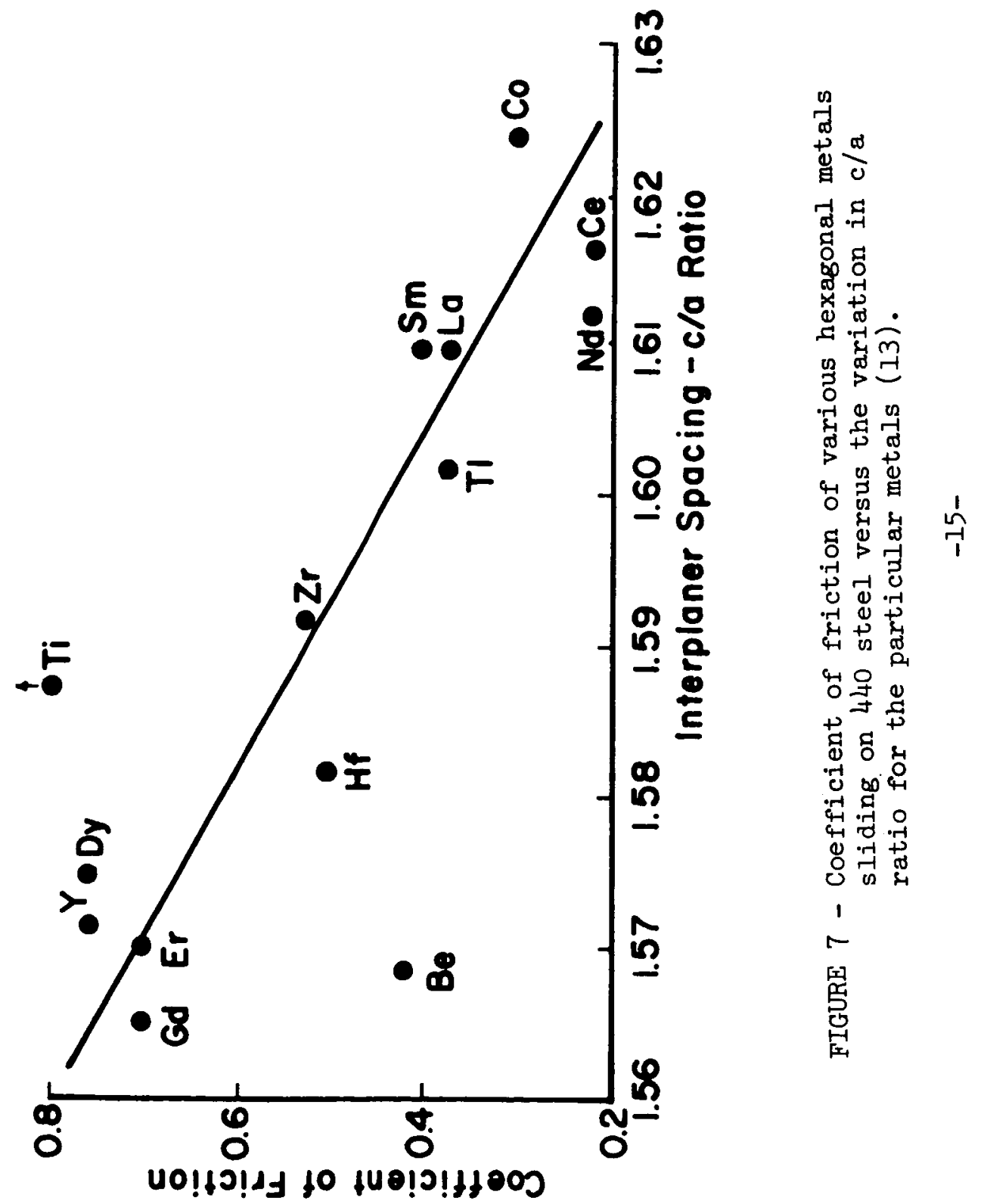




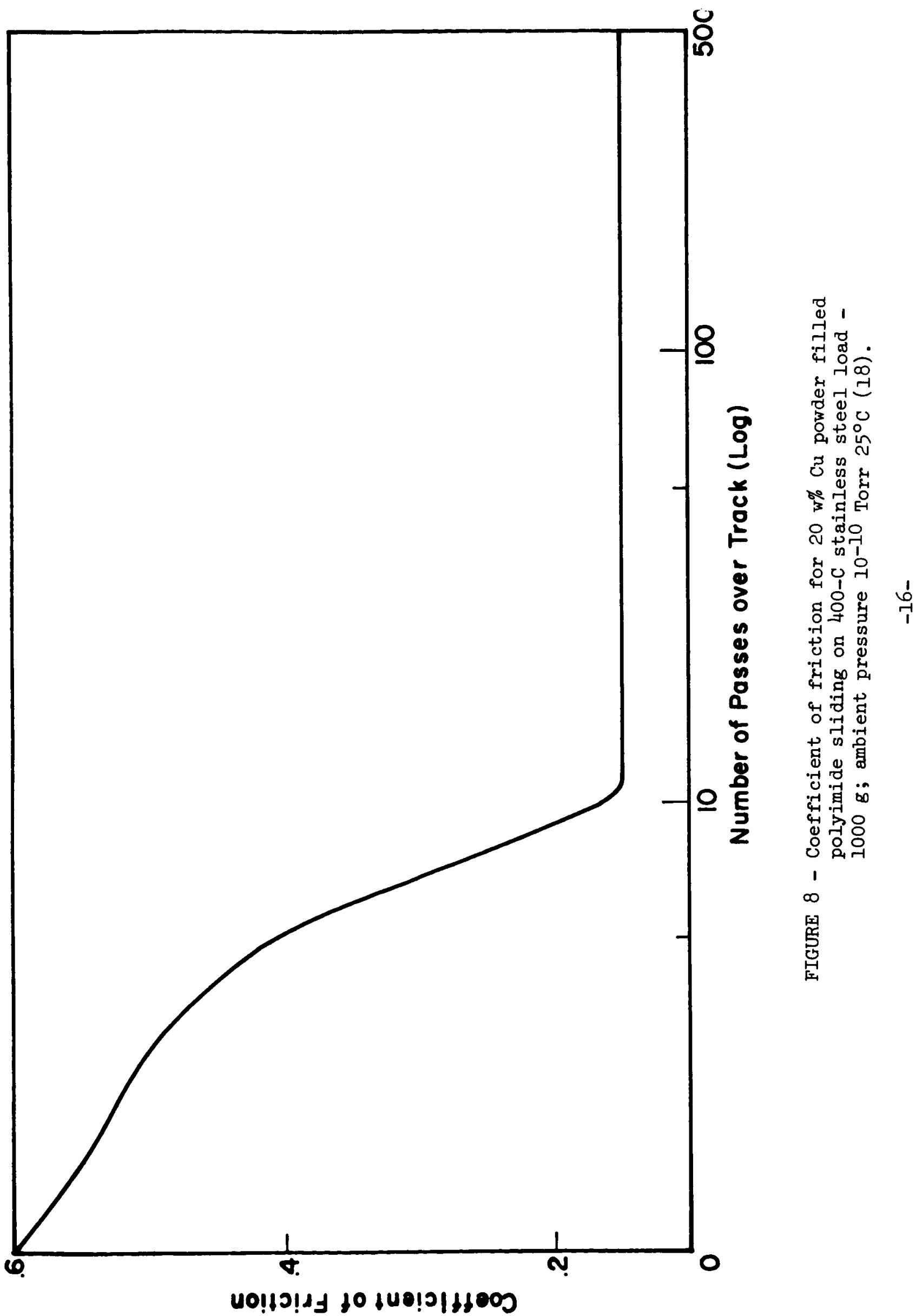


of passes over the same track (18). The initial coefficient of friction of polyimide on 440-C steel at $10^{-10}$ Torr was 0.6 and as the track became contaminated with polyimide by the transfer of polyimide film, the friction coefficient aropped to about 0.15 .

Liquid metals behave in a similar manner as shown in the study of nickel sliding on nickel with various liquid metal lubricants at $10^{-9}$ Torr which is illustrated in Figure 9 (19). Digressing for a moment, it now should be evident why the role of water, or other oxidizing agents, is so important in the investigation of frictional phenomena; in effect, a surface saturated with these components presents only molecular bonding fields to the mating surface rather than the stronger short range ionic or metallic bonding fields. When only the latter are involved the friction study seems to degenerate to a dynamic fracture problem in which the deformation and fracture characteristics of the two surfaces play the significant role. Whether or not the previous in vacua studies have achieved this end to complete perfection, is not known since secondary in situ experiments on the interacting surfaces was not performed. Had this been done, the degree of oxide contamination and its role in the friction experiment could have been discussed.

In an attempt to reduce the frictional force into its various constituents, two groups have evolved: those concerned with the deformation properties of the surface, those irregularities always present on real surfaces; and a second group entertaining the problem of achesion, that is, the materials problem of friction. The relationship between adhesion, or the attraction of two surfaces to each other, and friction is most unique since the term adhesion has usually been concerned only with the static system while friction is a kinetic process yet during the observation of adhesion phenomena a dynamic system must be employed. For example, 


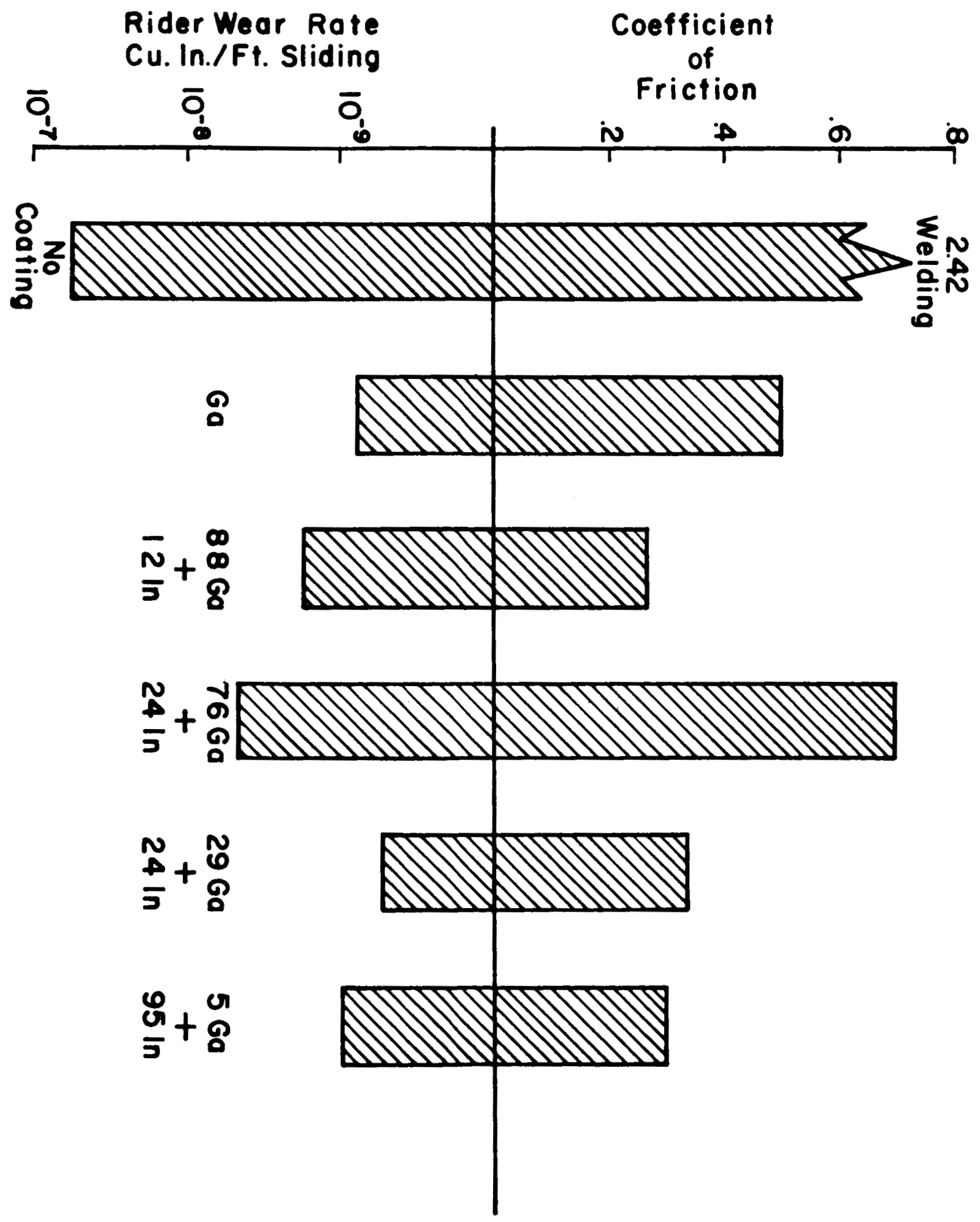

FIGURE 9 - Effect of various coatings on friction and wear of nickel at a velocity of $5 \mathrm{ft} . / \mathrm{min} ., \mathrm{a}$ load of $1000 \mathrm{~g}$; and periods of $1 \mathrm{hr}$. (Pressure: $10^{-9}$ Torr). 
in most adhesion experiments two surfaces are brought together by a normal force with or without a degree of tangential motion depending on the particular experiment, then to measure the "degree" of adhesion an opposite force is applied to the junction and the force required to fracture the junction is measured. The results are usually published as the coefficient of adhesion $(\alpha)$ which is the ratio of the force to separate to the force to make the particular junction with no regard for tangential motion. Hopefully, the desire has been to relate the coefficient of adhesion to $\mathrm{k}_{\mathrm{a}}$ of Equation (2); but, before we consider this possibility let us firstly review some of the details which have been described during the various adhesion studies.

Adhesion in Solid Systems

The study of adhesion seems to be limited by the same bounds as that indicated for friction, which is discussed in the more recent treatises on the subject (20-23), to cite a few. At the atomic and molecular level, the subject is usually treated under adsorption and desorption phenomena and in the organic, and occasionally in the inorganic, realm as wetting and dewetting phenomena. Generally, the gaseous and liquid interactions with other materials seems to have received the bulk of the study while adhesion between crystalline solids has been quite neglected until only recently. As indicated by Fowkes' lecture (24), the knowledge of the interactions between liquids and solids has achieved a reasonably high degree of sophistication incorporating ratios of particular interfacial fields to explain the various phenomena, which has been observed. In case of solidsolid systems, on the other hand, the state of affairs is not nearly as straightforward since the system under investigation involves two rigid bodies, neither of which has a simply-defined surface energy or physical geometry which must be brought into mechanical contact without gross 
perturbation of the surface layers under conditions of controlled contamination. Since the purpose of the ideal solid adhesion experiment is to provide a rational interpretation to that component in the frictional force equation, and its variation under the effects of contaminants, temperature, stress, translational motion, etc., the investigator is faced with the age old problem of reducing all of the possible variables in the system to a minimum before investigating each one individually. In the case of metals the problem becomes most evident; for example, silver does not weld noticeably to second silver surface in air under light loads, nor in high vacuum or ever in ultra-high vacuum after being baked therein to $450^{\circ} \mathrm{C}$. An atomically clean surface of silver, on the other hand, will adhere to a second clean silver surface with a junction strength equivalent to that of annealed silver as would be expected, particularly if we can visualize an ideally reversible cleavage experiment on a silver bar. The simple set of experiments just described points up two of the most important points revealed, thus far, in solid adhesion investigations: firstly, the degree of adhesion can only be measured as a fracture phenomena; and, as such, cannot be simply correlated to the thermodynamics of the system, e.g. work of adhesion, etc., due to the inability to analyze the plastic deformation of the system in such terms. Secondly, the presence of a monolayer or more of contamination at an adhesion junction, either by accident or purpose, can grossly affect the observed fracture data.

In-order to reduce these qualitative experimental observations on silver, cited above, into a quantitative experiment in which the unperturbed interfacial strength can be correlated with those under varying conditions of temperature or interfacial contamination, an apparatus was designed to permit two metal surfaces to be brought into contact with loads, which could be reversed and varied from 0.01 gms to about 2 gms (25). 
Since a reference point for such a study is necessary and the fact that oxides adhere to metals as well as metals to metals, the various equipment was installed in an ultra-high vacuum envelope which was operated in the range of pressures to $10^{-10}$ Torr and capable of performing the various metal cleaning steps of argon ion bombardment and electron beam annealing, as suggested by the various low energy electron diffraction (LEED) investigators. Since the investigation in itself is surface destructive if an adhesion weld occurs, indications early in the research program suggested that the in situ use of a LEED experiment or a field ion microscope experiment would provide positive proof of the surface cleanliness of one surface while multiplying the complexity of the experiment almost beyond reach; therefore, such an approach was avoided. In the interim, investigations on the feasibility of the utilization of contact resistance to indicate the exact area of contact junction produced some rather interesting results. The results seemed to suggest that by means of this measurement a semi-quantitative analysis of the changes taking place in the interface region, i.e. about $0.1 \mu^{2}$ in area, during force variation and/or contamination could be investigated.

Briefly, the system used for this investigation as described in detail by Johnson and Keller (25), consisted of a 40 x $300 \mathrm{~mm}$ pyrex adhesion cell (A) attached to a $I^{\prime \prime}$ ultra-high vacuum valve $(H)$ and, thence, to the vacuum system, as shown in Figure 10. The adhesion cell, valve, and first liquid nitrogen trap were baked-out during each experiment at $450^{\circ} \mathrm{C}$ for at least 10 hours. After bakeout, the degassing of the titanium sorption pump and other filaments, and the cooling of the first liquid nitrogen trap, the minimum pressure observed in the adhesion cell was $5 \times 10^{-10}$ Torr, as measured by the NRC Redhead gauge (D) mounted adjacent to the specimens. The titanium sorption pump (E) consisted of a 


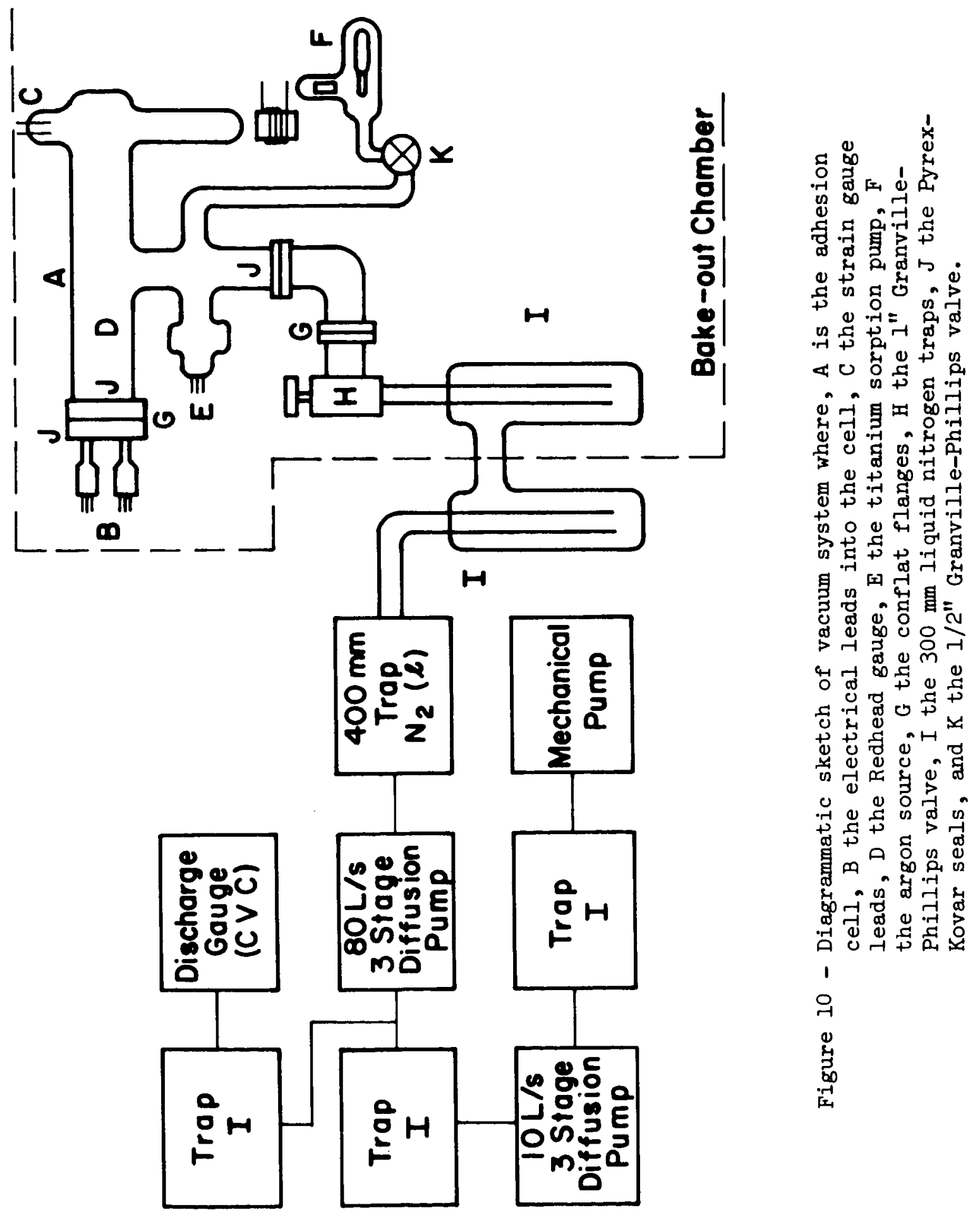


helix of $0.010^{\prime \prime}$ titanium wire closely wrapped over $0.015^{\prime \prime}$ tungsten wire.

The torsion beam and adhesion samples are shown in Figure 11 . Both were supported by three $5 \mathrm{~mm}$ stainless steel support rods heliarc welded to a stainless steel Conflat flange plate attached to the cell at $(\mathrm{J})$ in Figure 10. The rods also served as supports for the sample electrical leads within the chamber, which were all insulated with recrystallized alumina tubing and exited the cell by standard Kovar through-seals at (B), Figure 10. The torsion beam was also constructed of alumina tubing and was supported at its center by a stainless steel connector which served as a bearing for the torsion beam as it rested on a $0.010^{\prime \prime}$ tungsten wire under tension between the two $5 \mathrm{~mm}$ stainless steel supports.

The iron slug ( $F_{1}$, Figure 11) fixed to the end of the torsion beam, was used in conjunction with the external permanent magnet (c) to affix the position of the indenter with respect to the sample plate at a finite distance from actual contact. The strain gauge (G) mounted on the torsion beam, supported a second iron slug, $F_{2}$, which interacted with the field of a solenoid $(\mathrm{L})$. Thus, as the current in the solenoid, monitored by the calibrated variable resistance $(J)$ was increased, the torsion beam was moved into contact with the fixed sample (at point A) and a normal force placed on the sample plate due to the indenter. The force of shearing the magnetic flux between the iron slug, $F_{I}$, and the magnet (C) before contact, and the force of contact of the indenter (B) with the fixed sample plate (A) were measured by the $0.00095^{\prime \prime} \times 6 "$ nude straight constantan wire strain gauge, whose output was monitored by a Sanborn Transducer-Amplifier, Model 312. Prior to each experiment the balance system was calibrated in air throughout the range of operation, i.e. $0-2.0$ grams, and was found to have a sensitivity of about \pm 0.010 grams. The contact resistance between the indenter and plate was measured 


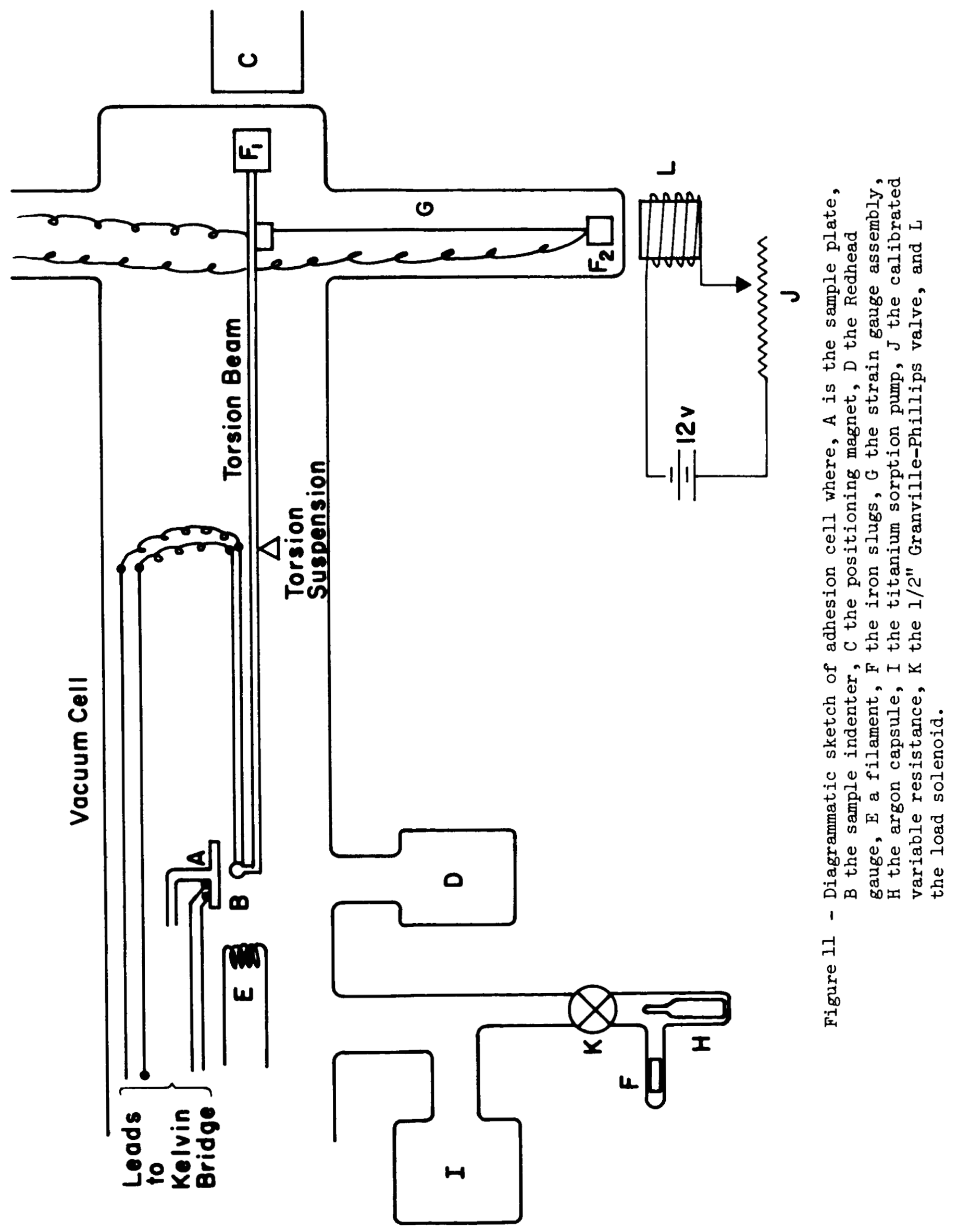


with a Precision Kelvin Bridge in confunction with a Nanovoltmeter used as a null detector by the standard cross-wire configuration. A source was used such that the potential drop across the contact resistance was approximately 0.3 millivolts, which should yield negligible temperature rise at the contact region due to current flow (26). Such an arrangement enabled the resistance to be measured when within the range of zero to one ohm, with an accuracy of 3-4 figures. The resistance circuit was calibrated with a 0.01 ohm NBS standard resistor prior to each run.

The torsion beam arrangement was designed in the above way in order to obtain, as nearly as possible, pure normal loading. Thus, shear deformation of the adhesion specimens was reduced to a minimum during test cycles, the only tangential motion being imparted to the specimens by unavoidable normal laboratory vibrations. The effects of these could only be observed under extreme light specimen loading and non-adhesion conditions, when instability of the contact resistance occurred.

The normal operating procedure involved placing the samples in the system and evacuating to a pressure below $10^{-5}$ Torr, at which time the bakeout cycle was imposed, as previously mentioned, to attain an ultimate pressure of about $5 \times 10^{-10}$ Torr. At this time the ultra-high purity argon, obtained from Airco Company, was admitted to the leak system by breaking the capsule break-off tip. The argon was then admitted to the cell to a pressure of about $10^{-4}$ Torr, and argon ion bombardment of each surface initiated by placing a D.C. potential of about a kilovolt between the filament (E), Figure 11 , and the surface to be cleaned. During the cleaning operation, which amounted to a total of at least three hours for each surface, a small nickel shield was moved into place (via magnet) to completely shield the surface not being cleaned from contamination by sputtered material. After bombardment, a substantial sputtered deposit 
on the cell walls attested to the fact that a considerable amount of surface material was removed from each sample. Upon completion of the argon ion bombardment phase, the system was evacuated and sample annealing initiated. Electron bombardment from the filament (E) was used to heat the sample for argon degassing and sample anneal.

At certain points throughout the whole of this evacuation and surface cleaning process a series of adhesion cycles were performed at room temperatures by slowly bringing indenter (B) into contact with (A), by reducing the variable resistance $(J)$. The values of $(J)$ and the deflection of the transducer amplifier, due to the strain gauge, were noted concurrently at discrete intervals until sample contact was made, when contact resistance measurements were also performed at each new adjustment of $(J)$. The load on the adhesion couple was then further increased to a predetermined level and then reduced by increments until contact was broken. Contact make and break were immediately indicated by a closed and open circuit in the Kelvin Bridge. In this way the loading and unloading processes were monitored by at least ten concurrent contact resistance, force, and solenoid circuit resistance measurements during each adhesion cycle. The peak loads employed were usually 0.3 and 1.5 grams under a very low impressed voltage such that contact heating could be considered nil (26). Under various contaminant conditions the junction resistance can vary from infinity (insulating oxide layer) through nonohmic, or semiconductive, to pure ohmic as thoroughly discussed by Holm (28). Since the relationship of contact resistance to the contact area is not simple, the contact resistance data cannot be utilized in an absolute sense; however, the data are most valuable in a relative sense for comparing the degree of interference to electron flow through the contact region, e.g. contamination, from one stage of contamination to another 
as well as studying the effect of contact force on the change in contact area in one experiment.

Let us examine the results of one adhesion cycle (contact, to maxi-

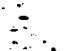

mum load, to fracture) under conditions in which the surfaces of silversilver could be considered very nearly atomically clean (Note: the absolute sense is not justified since no internal measurement such as low energy electron diffraction techniques were employed to justify absolute cleanliness). The adhesion cycle illustrated in Figure $12 \mathrm{a}$ shows the effect of variation of the solenoid current (as circuit resistance), which tended to rotate the torsion balance arm in order to bring the silver samples into contact versus the contact force strain gauge reading. The moment of contact of the samples is observed as the first contact resistance value as shown in Figure 12b. The samples used to produce these curves were rigorously outgassed at $10^{-10}$ Torr and then subjected to rigorous argon ion cleaning at a current density of at least $500 \mathrm{amps} / \mathrm{cm}^{2}$ at one kilovolt for about one hour in spectrographically pure argon. Since these curves are representative of the over five hundred tests to date of which a major portion of the curves did demonstrate adhesion, we feel that a curve of the shape shown in Figure $12 b$ can be used as a criteria for adhesion. That is, as the load is released the contact resistance is virtually constant until, or very close to, the point of fracture. This observation indicates that released elastic stresses, as suggested by certain authors (1), do not play a major role in the fracture of silver-silver ( $\mathrm{Ag}-\mathrm{Ni}$ or $\mathrm{Cu}-\mathrm{Ni}$ ) adhesion junctions (recent tests show that this is also true in the titanium-titanium and molybdenum-molybdenum system where testing is all in the elastic range). In comparing an adhesion case to one in which contamination prevented adhesion in the silver-silver system, two factors were immediately evident in the lightly loaded systems under test. As shown in 


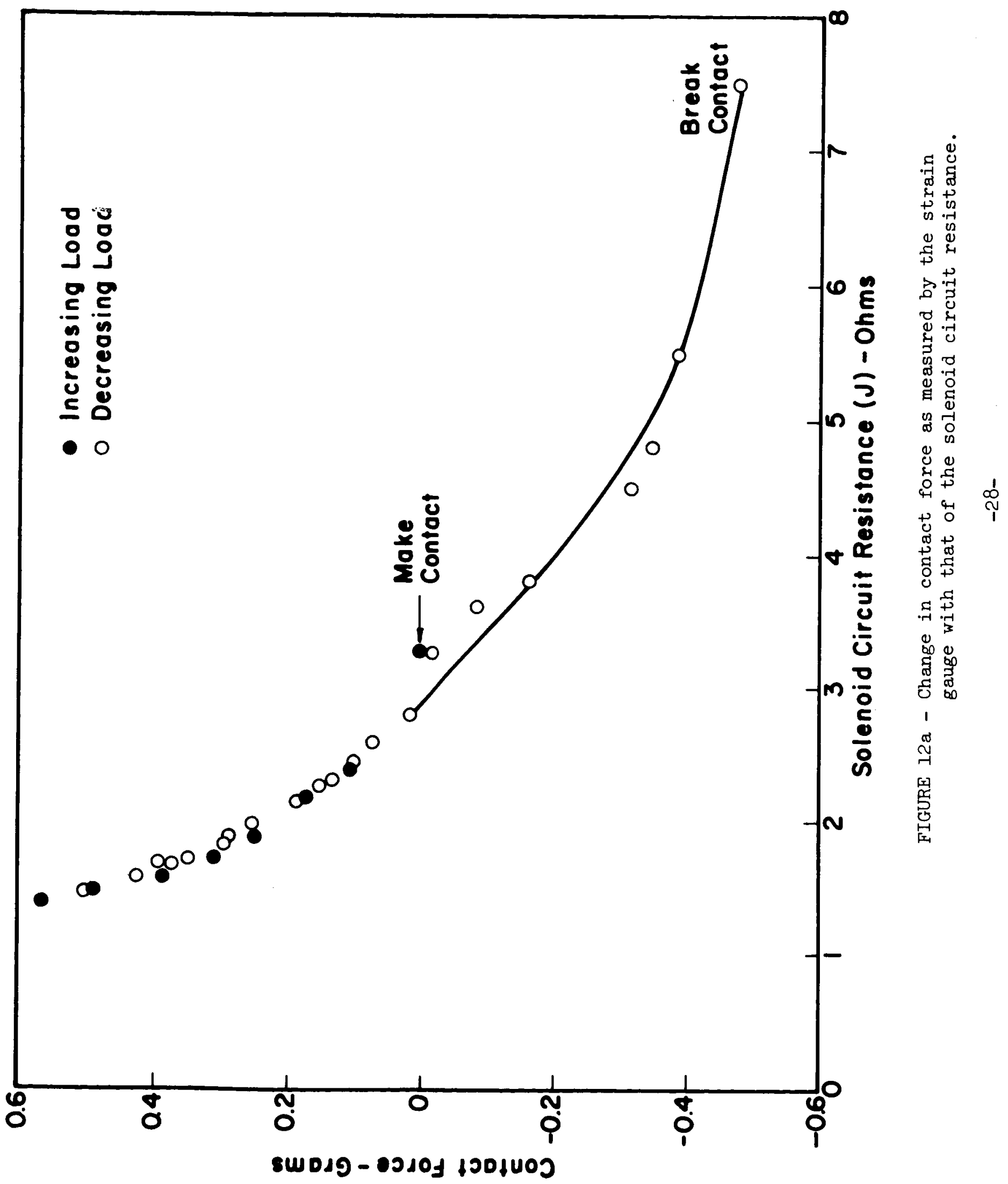




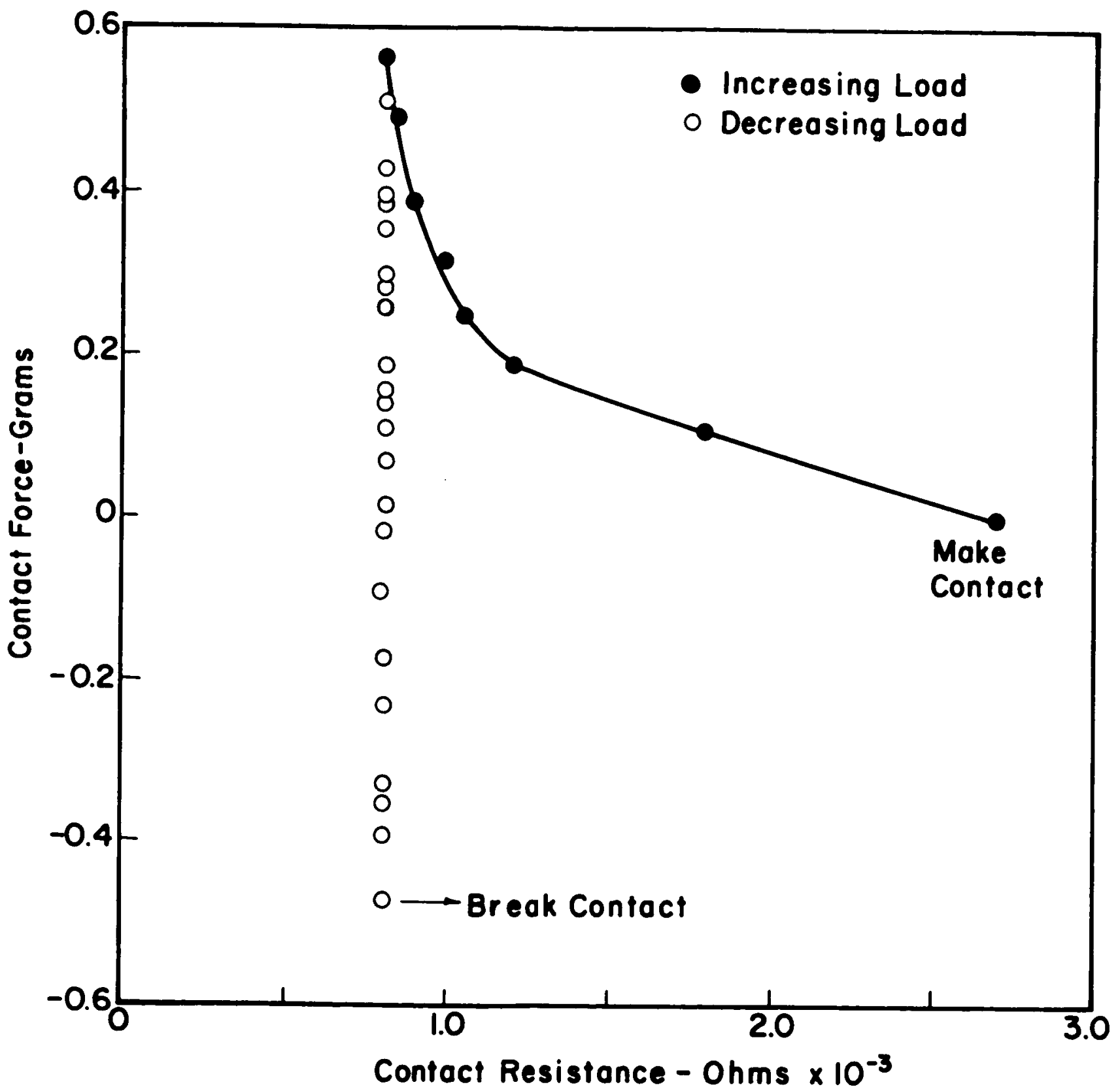

FIGURE $12 b$ - Change in contact resistance of a silver-silver couple with contact load for surfaces which are Ultra clean and demonstrate adhesion. 
Figure $12 \mathrm{c}$ a substantial load of approximately $0.3 \mathrm{gms}$ was required to permit a stable resistance reading, and after peak loading the released elastic stresses apparently decreased the contact area, such that the contact resistance superimposed along the loading curve returning to the point of instability. Whether or not this represents the ability of the contaminant oxides along the interface to fracture with ease or their total inability to form an adhesion junction, cannot be readily ascertained from this experiment. What is important is that a distinction between weak and strong interactions can be made through a secondary measurement, which provides some insight into the status of the interface. The difference in contact loading values versus contact resistance values should be noted between Figures $12 b$ and $12 c$.

To explore this fact further, Figure 13 illustrates the data from Figure $12 b$ plotted as a log-log relationship with a curve for the variation in contact resistance of crossed silver wires tested in air. Of significance is the fact that for a constant load the removal of the contaminant layer from silver reduces the contact resistance by a factor of six, which is significant, since the precision with which this difference can be measured is about three or four significant figures without taking any special precautions. The variation of contact resistance with load as the cleaning process proceeds is also of interest, for it demonstrates changes that take place in the contact region as the ambient conditions change. Figure 14 shows the variation in contact resistance of the silversilver system versus load beginning with etched silver samples in air, and comparing these values with those in vacuum, and, thereafter, with those in vacuum after bake-out $\left(450^{\circ} \mathrm{C}-20\right.$ hours) and $900^{\circ} \mathrm{C}$ anneal at $10^{-10}$ Torr, and finally after argon ion cleaning. No adhesion was indicated until after the preliminary degassing process was completed, and the junction 


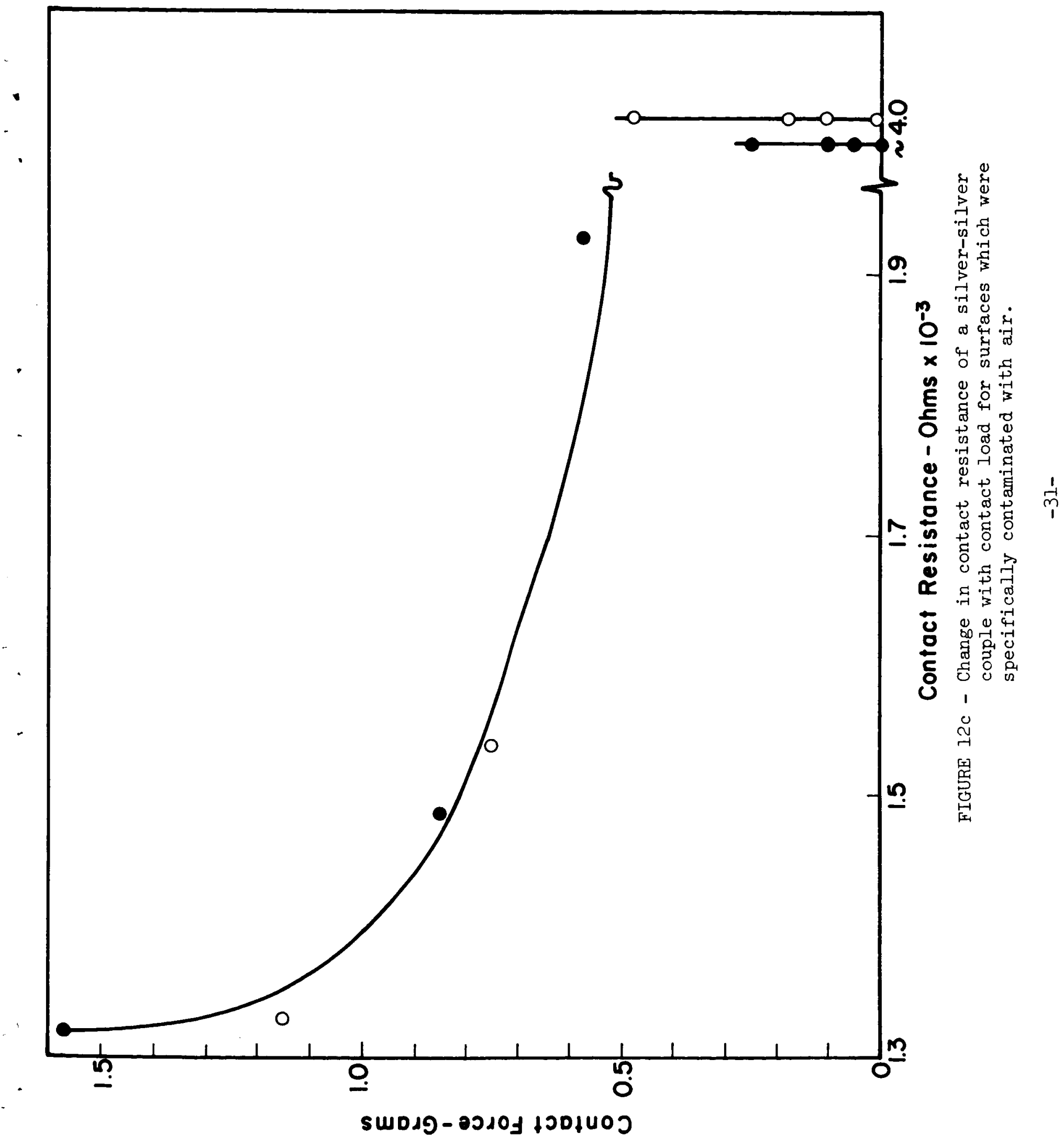




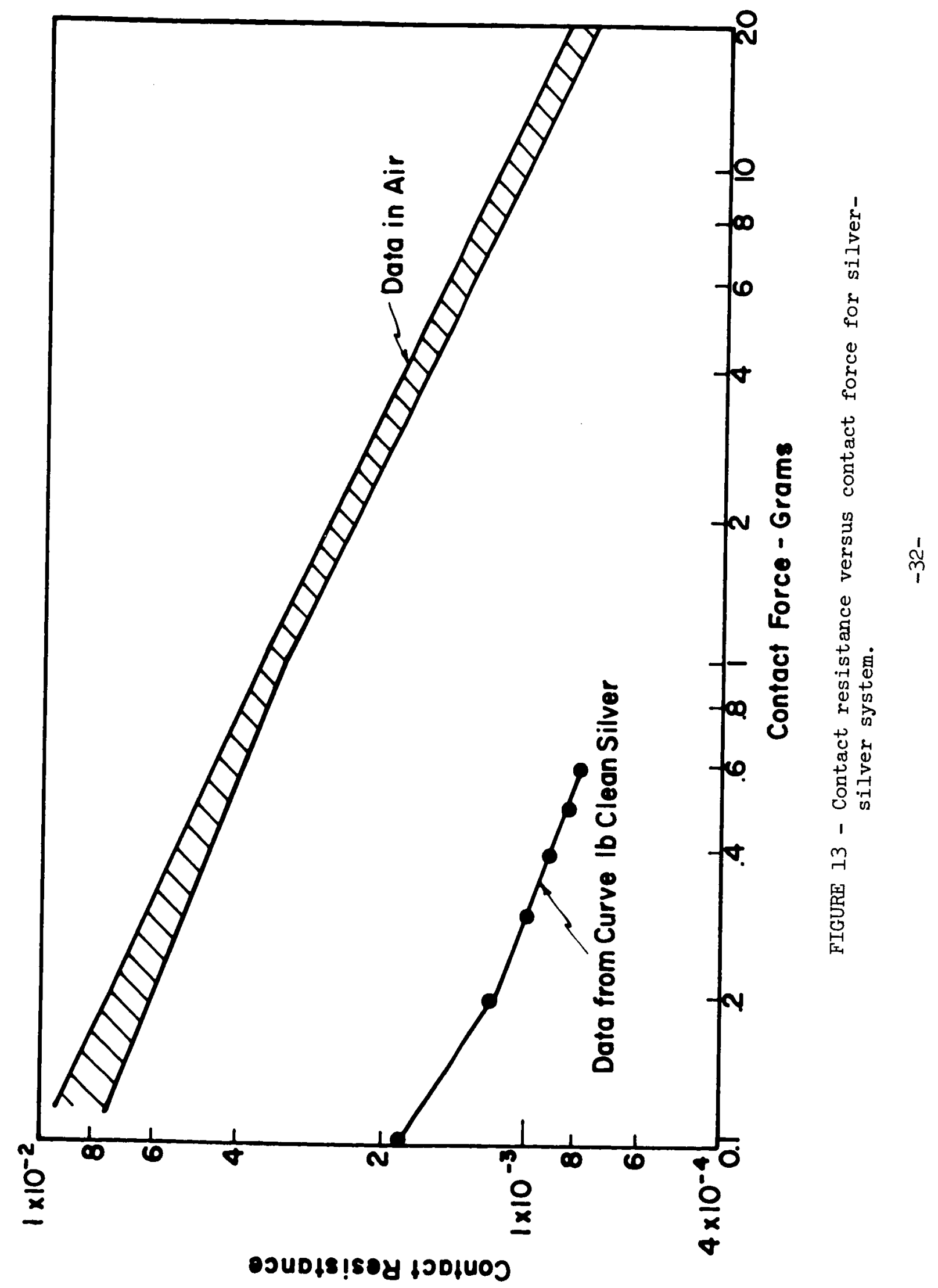




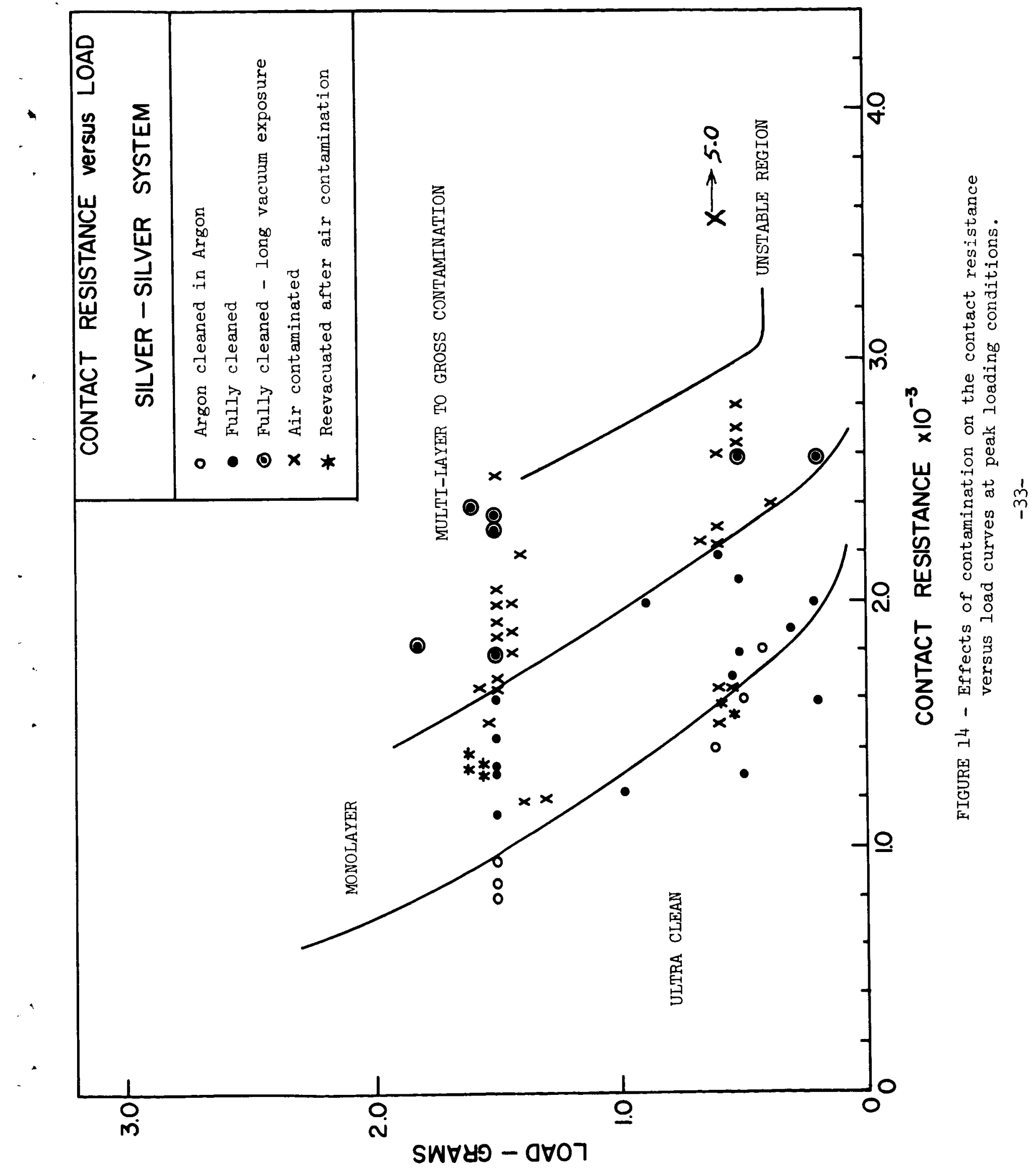


strength only reached the strength of silver only after argon ion cleaning. The contaminant films present on silver seem to be of two distinct types, those which are partially removable by vacuum and degassing techniques, and those which are only removable by rigorous ion cleaning techniques. The latter is supported by the observations of a very tenacious film on silver by Farnsworth and Winch (27) during their investigation of the work function of silver. Therefore, in a qualitative sense contact resistance measurements provide a simple method for the interpretation of boundary conditions during adhesion testing. With a large quantity of data one is tempted to investigate just how far these observations can be interpreted; and in this line of thought, Figure 15 was developed. By expanding the coordinates of Figure 14 one can examine the data from very clean surfaces and adsorbed films in detail. Again, each point represents the minimum contact resistance observed in a particular adhesion cycle under various conditions of contamination. The curves have no real significance, but only act as a guide line to the surface conditions that might be expected by the particular experimental conditions. Whether or not the points in the ultra-clean region are truly the minimum values of contact resistance, can only be ascertained by either a separate experiment (low energy electron diffraction) or a multitude of data from separate experiments on silver. Figures 16 and 17 are similar to Figure 15 except that dissimilar couples were used. Again, the contact resistance reflects the nature of the film, but only in a qualitative fashion.

The necessity of maintaining a qualitative sense to the analysis with regards to the absolute contact resistance data discussed above can be justified briefly. The resistance $(R)$ to current flow across a metal contact interface can be represented by

$$
R=R_{0}+R_{M}
$$




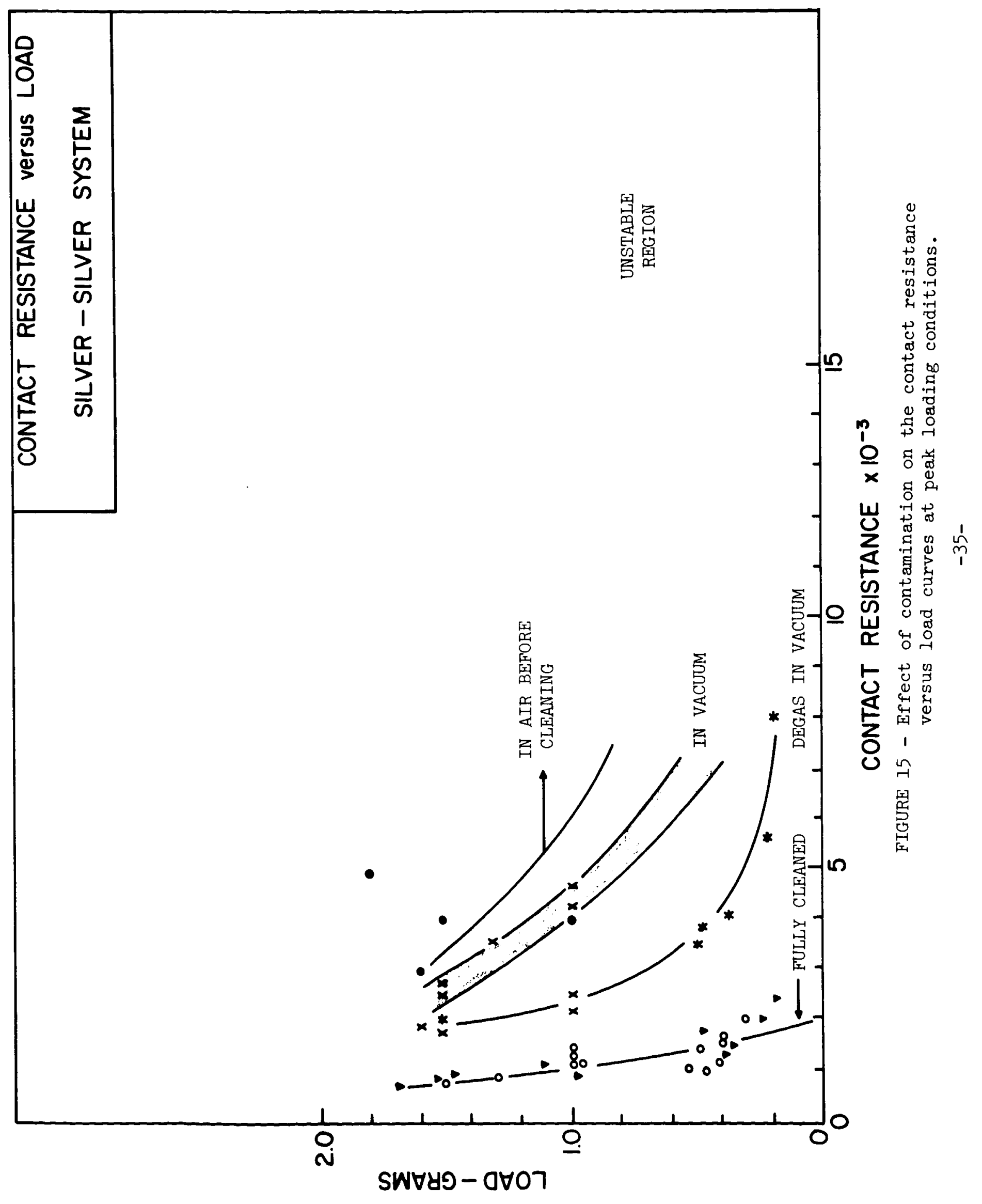




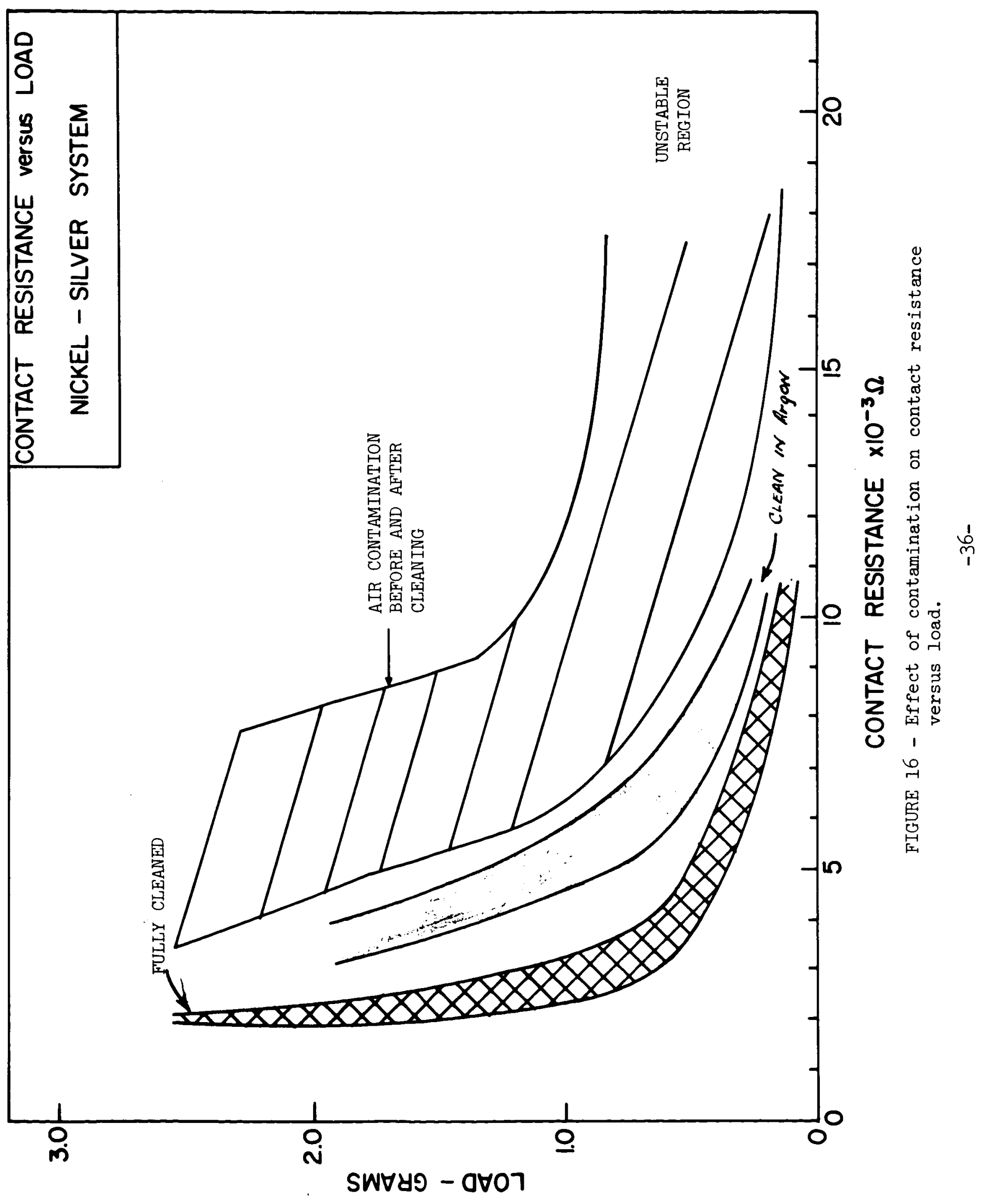




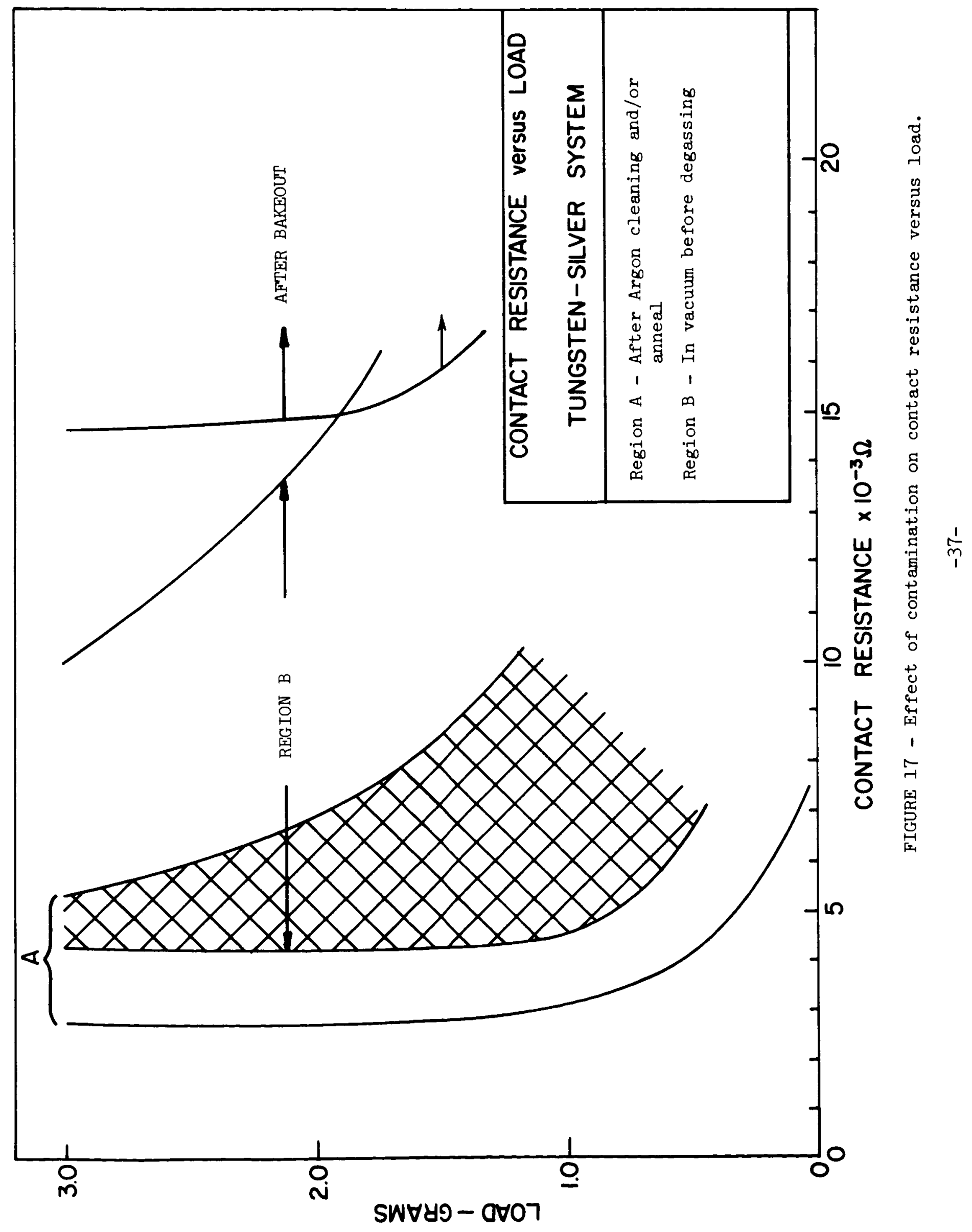


where $R_{0}$ represents the resistance due to an oxide, or contaminant film, which, if thick enough, can be the controlling factor, i.e. act as a pure insulator. $R_{M}$ is the constriction, or contact resistance due to the compression of the potential lines of force in a metallic contact from one macro-body through a very small diameter contact back to a second macrobody. The variation of $R_{0}$ with surface cleaning ought to be from infinity, or pure insulator, through semiconducting for extremely thin films to zero for atomically clean surfaces. The variation, however, is probably affected by the contact pressure as well as the area change during load variation, and is probably a most complex function of load and contaminant chemistry. The theoretical nature of $R_{M}$ was developed by Holm (28) and has recently been re-evaluated by Greenwood (29), who showed that $R_{M}$ is a function of the metal conductivity $(p)$ and the number $(n)$ of metal junctions at the interface by the equation,

$$
R_{M}=\rho\left(\frac{1}{\pi n^{2}} \sum_{i \neq j} \frac{1}{r_{i j}}+\frac{1}{2 \sum_{i} a_{i}}\right)
$$

where

$$
\begin{aligned}
& r_{i j}=\text { is the distance between } n \text { metal contacts } \\
& a_{i}=\text { radius of the } \underline{i t h} \text { contact }
\end{aligned}
$$

Since a metal contact region may have any number of asperities of various diameters in real contact at any variation in separation, the equation, in effect, indicates there is little hope for reasonable analysis from basic principles. Whether or not an empirical relationship can be established, based on a simple model (28), remains to be seen. The linearity of the loading data, shown in Figure $12 \mathrm{~b}$ when it was plotted on a log-log curve (Figure 13), indicates Holm's single contact relationship for clean surfaces,

$$
R_{N}=\frac{p}{2 a}
$$


where

a = the contact radius which, in turn, is a function of the deformation properties of the material versus load

is a reasonable approximation and some hope that an empirical relations may evolve. Further evidence of this was established when the log of the contact resistance versus the log of the load of the individual adhesion cycles were plotted and, for the most part, produced straight lines with a slope lying between $\left(-\frac{1}{3}\right)$ or $\left(-\frac{1}{2}\right)$. The minus one-third slope represents an area variation due to elastic deformation of the metal and the latter due to plastic deformation (28). The intercepts of these lines were also of the right order of magnitude, but displaced between 10-40\%, which was probably due to contamination or multi-contact points and did not seem too far removed, considering the complexity of the proposed mathematical relationships.

Exactly where does this place the contact resistance type of experiment in the realm of adhesion testing? Firstly, let us examine the coefficient of adhesion parameter obtained from the silver-silver experiments with the realization that this parameter assumes that the elastic and plastic deformation, in forming the contact junction, was equal to that during the fracture of that junction. Of course, one bears no relationship to the other, so the numbers are not wholly significant, but if accepted in a relative sense, they do offer a comparison. Table 1 illustrates the extreme effect of the atmosphere on the relative strength of the adhesion function. The air cited in the table was dried over silica gel for several days prior to use; however, in exploiting this behavior further, individual pure gases, i.e. nitrogen, oxygen, hydrogen and water, are in the process of being examined to ascertain which has the greater effect as a barrier in the adhesion process. The use of various chemical 
TABLE I

APPROXIMATE COEFFICIENTS

FOR

ADHESION *

Pressure Torr

1. $10^{-10}$

2. $\sim 4$ min argon

3. $8 \times 10^{-9}$

4. $\sim 10^{-4}$

5. 760

6. $5 \times 10^{-7}$
Conditions

$$
\text { before cleaning }
$$

immediately after cleaning

air inleak from $10^{-10}$ Torr after cleaning

air inleak from \#3

air inleak from $\# 410^{-10}$ Torr

after atmosphere exposure(s)
Average $\alpha$ (at least 10 runs)

\section{$2.2 \pm 1.2$}

$0.33 \pm 0.16$

$0.19 \pm 0.17$

$0 \neq$

$0.16 \pm 0.08$

* cross wire loading $0.01-2.0$ grams at $25^{\circ} \mathrm{C}$

fminimum detectable $\alpha=0.005$ 
agents was also proposed in an attempt to optimize the process. As the various atmospheres are examined the contact resistance data is also accumulated during the test, which will: a) permit a qualitative picture of the fracture process, e.g. Figure $12 b ; b)$ some indication of the relative thickness of the film by contact resistance value; and, c) hopefully, a reasonable correlation of the resistance values with area will be achieved which, in turn, will provide an accurate knowledge of the real contact area. If a knowledge of the real contact area can be obtained, the testing data can then be reduced to an absolute scale and related to the mechanical properties of the material in test.

The development of the adhesion-contact resistance experiment, if nothing else, permits three independent simultaneous indications of the presence or absence of adhesion, and permits two independent measures of the strength of the junction formed. On this basis, we feel that the reliability of metallic adhesion data will be increased; and, as a side effect, some insight into the behavior of contaminant films will be gained. Furthermore, as the confidence level of adhesion data is expanded, two very fruitful areas of investigation are immediately available, namely, the effect of alloy constituents on metallic adhesion and the effect of specific chemical agents, either in metal solution or as gaseous contaminants on metallic adhesion can be investigated in a rather quantitative manner. Since the above experimental data has related the adhesion strength of a system to the fracture strength of the weaker component, the utilization of surface chemistry as a basis for research direction in adhesion studies would seem the most fruitful, particularly, if specific release agents, i.e. low shear stress boundary agents, were desired to reduce the adhesion component in friction systems. 
Further evidence that the presence of a monolayer of adsorbed gas adsorbed along the interface has a gross effect on the strength of that interface was recently presented by Aldrich for liquid metal-solid metal systems $(30)$.

The Adhesion-Friction Relationship

Since the purpose of this investigation was to examine the behavior of adhesion phenomenon for its ultimate incorporation in the mechanism friction, the utilization of the special friction case in which the plowing component is neglected should be justified; and, as a consequence, Equation (5) is

$$
F=A s
$$

operative. The broad usage of this equation, at first, does not seem justified since the terms are, according to the adhesion data presented above, most difficult to tie down. The real area, for example, in a friction experiment should include not only the regions of real physical contact but all of those regions which, due to their proximity to the second surface, are interacting by means of long range dispersion fields (3I) and medium range electromagnetic fields. In lightly loaded high surface area systems, these fields may well be as significant as the short range bonding forces. In regards to the short range forces, the existence of metal:non-metal as well as non-metal:non-metal interactions also cannot be neglected. Similarity in the lubricated systems, the molecular fields resulting in the cohesive energy of the lubricant cannot be neglected. Therefore, in the consideration of the real area of contact all possible regions and their attractive forces must be considered; and, as a consequence, the extreme simplicity of the "dry" metal friction experiment becomes most evident.

The shear parameter in the equation is usually reserved for that of 
the component with the weakest shear strength in a sliding system; however, this, too, must also be most complex since it relates directly to those junctions described above. In other words, a few very small diameter islands of metal-metal contact in a large surface area of real contact might be weaker than the shear strength of the mass of lubricant separating the two apparent areas of contact. In such a case, the shear strength of that portion of the lubricant involved in the true contact region ought to be considered as that providing the resistance to flow plus some added factor to include the shear strength of the islands of metal-metal bonds. Probably a more realistic expression for the force of friction in this simple case might be written as a series of summations, such as:

$$
F=s_{1} \sum A_{1}+s_{2} \sum A_{2}+s_{3} \sum A_{3}+\ldots
$$

with the limitation that if any one $s_{i}$ exceeds the shear strength $\left(s_{j}\right)$ of the region immediately adjacent to that in question, $\mathrm{s}_{\mathrm{g}}$ should be substituted for $s_{i}$. This would be expected to occur under such conditions in the bulk, i.e. the weaker material, rather than in the interface. The $s_{1}, s_{2}, s_{3} \ldots$ values represent the shear force contributions required in the fracture of metal-metal, oxide-oxide or lubricant-lubricant junctions as well as dispersion and other field effects such as magnetism, that is, each particular component contributing to the overall effect. Since the coefficient of rolling friction is usually a much smaller value than the corresponding value of sliding friction, one might further suspect that the respective tensile values for these same fracture systems might be considerably smaller. The latter point seems to be supported by the observation (1) that tangential stresses applied to a static adhesion tests generally increase the observed adhesion strength. The complexity developed in the equation above seems, at first, to 
be insurmountable; however, if this equation were applied to a lightly loaded, boundary lubricated system in which all real areas of contact involved only lubricant the complex equation reduces simply to Equation (5) in which the shear strength of the lubricant is significant and the real area of contact that region bearing the load. As the load is increased, however, and asperities from each of the surfaces do come into contact, the other parameters must become operative and included in the expression.

In the case of friction between wood and glass the terminology "adhesion", or "cold welding", becomes a misnomer, particularly as it was applied to metallic systems; but, in fact, remains a real phenomenon in the sense that interacting molecular fields of the dispersion type are still operative; and as motion proceeds, provides a resistance to that motion. This phenomenon is quite similar to the restriction to rolling experienced by a steel ball attempting to roll down the inclined plane, which consists of one pole of a magnet only on a much smaller scale. Consequently, in the application of all of the force fields available to adhesion phenomena and after the fact analysis seems to be probable for most friction phenomena. What does remain to be clarified is a far more detailed picture of the interaction of two static parallel surfaces from distances of separation of one micron down to non-equilibrium contact and the effect of the normal variables on this system.

\section{CONCLUSIONS}

Recent investigations in the field of metallic adhesion seem to support the general adhesion theory for the mechanism of friction in that as a better understanding of the adhesion phenomenon is achieved an improved correlation seems to exist. Apparent inconsistencies in the adhesion theory of friction which have been reported in the past can, for the most part, be explairred through the course of new data in the fields 
of surface physics and chemistry as well as more accurate adhesion data. The inaccuracy of adhesion data distinctly refers to the inability of the author to define the interface system under investigation in detail in terms of monolayers and not the technique that was employed. For, as has been illustrated in the adhesion investigations of $\mathrm{Ag}-\mathrm{Ag}$ and other systems (Mo-Mo, Ti-Ti, Cu-Ni, Ni-Ag, In-Al), the presence of a few monolayers of contaminant may distort the data, and in turn the interpretation, most significantly. Since specific contamination seems to be the only barrier to metal-metal adhesion and the forces of adhesion can occur between any pair of materials in varying degrees up to separation distances of one micron, all interfacial force fields ought to be considered when the adhesion component in the frictional force equation is considered. In effect, this suggests that the physical contact area between the apparent surfaces of contact in a friction system is only a part of that area which ought to be considered in the final analysis.

\section{ACKNOWLEDGEMENT}

The author would like to acknowledge the financial support of the National Aeronautics and Space Administration for this program. 


\section{BIBLIOGRAPHY}

1) Bowden, F.P., and Tabor, D., "Friction and Lubrication of Solids", Clarendon Press, Oxford, Pt. I (1954), Pt. II (1964)。

2) Rabinowicz, E., "Friction and Wear of Materials", J. Wiley and Son, Inc., New York (1965).

3) Johnson, R.L. (Private Communication), Head Lubrication Section, NASA, Lewis Research Center, Cleveland, Ohio.

4) Antler, M., "Processes of Metal Transfer and Wear", in Mechanisms of Solid Friction, P.J.Bryant, M。 Lavik and G. Solomon, eds., Elsevier Publishing Co., N.Y. (1964) 181.

5) Cocks, M., "Interaction of Sliding Metal Surfaces", J.Appl.Phys., 33 (1962) 2152.

6) Steign, R.P., "Friction and Wear of Single Crystals", in Mechanisms of Solid Friction, P.J. Bryant, M. Lavik and G. Solomon, eds., Elsevier Publishing Co., N.Y. (1964) 48.

7) Goddard, J., and Wilman, H., "A Theory of Friction and Wear During the Abrasion of Metals", Wear, 5 (1962) 114.

8) Tabor, D., "The Hardness of Metals", Clarendon Press, Oxford (1951).

9) Westwood, A.R.C., and Kaindar, W.M., "Concerning Liquid Metal Embrittlement, Particularly of Zinc Monocrystals by Mercury", Phil. Mag., 8 (1963) 787.

10) Bryant, P.J., "Cohesion of Clean Surfaces and the Effect of Adsorbed Gases", 9th Nat.Vac.Symposium, Amer.Vac.Soc., p. 311 (1962) 311.

11) Buckley, D.H., Swikert, M., and Johnson, R.L., "Friction, Wear and Evaporation Rates of Various Materials in Vacuum to $10^{-7} \mathrm{~mm} \mathrm{Hg}$, ASLE Trans, 5 (1962) 8 .

12) Roberts, R.W., and Owens, R.S., "Boundary Lubrication of TitaniumTitanium and Titanium-Steel, Wear, 6 (1963) 444.

13) Buckley, D.H., and Johnson, R.L., "Influence of Crystal Structure on Friction Characteristics of Rare Earth and Related Metals in Vacuum to $10^{-10}$ Torr", ASLE Trans., 8 (1965) 123.

14) Buckley, D.H., and Johnson, R.L., "Friction, Wear and Adhesion Characteristics of Titanium-Aluminum Alloys in Vacuum", NASA TN D-3235 (January, 1966).

15) Buckley, D.H., and Johnson, R.L., "Marked Influence of Crystal Structure on Friction and Wear Characteristics of Cobalt and Cobalt-Base Alloys in Vacuum to 10-9 Torr", NASA TN D-2523 (1964).

16) Buckley, D.H., "Effect of Orientation on Friction Characteristics of Single-Crystal Beryllium in Vacuum at $10^{-10}$ Torr", NASA TN D-3485 (JuIy, 1966). 
BIBLIOGRAPHY (Cont'd.)

17) Buckley, D.H., "Influence of Orientation of Grains in Tungsten on its Friction Characteristics", NASA TN D-3238 (January, 1966).

18) Buckley, D.H., "Friction and Wear Characteristics of Polyimide and Filled-Polyimi de Compositions in Vacuum", NASA TN D-326I (Feb. 1966).

19) Kuczkowski, T.J., and Buckley, D.H., "Friction and Wear of Low Melting Binary and Ternary Gallium Alloy Films in Argon and Vacuum", NASA TN D-2721 (1965).

20) Eley, D.D., "Adhesion", Oxford University Press (1961).

21) Bickerman, J.J., "The Science of Adhesive Joints", Academic Press, N.Y. (1961).

22) "Contact Angle, Wettability and Adhesion", (Kendall Award Symposium) Adv. in Chem. Ser. No. 43, Amer. Chem. Soc., Washington, D.C. (1964).

23) Houwink, R., and Salomon, G., "Adhesion and Adhesives", Elsevier Publishing Co., Amsterdam, N.Y. (1965).

24) Fowkes, F., this Symposium.

25) Johnson, K.I., and Keller, D.V., Jr., "The Effect of Contamination on the Adhesion of Metallic Couples in Ultra-High Vacuum", submitted to J. Appl. Phys.

26) Williamson, J.B.P., "Significance of Non-Destructive Tests of Compression Joints", Proc. Inst. Elect. Eng., 109A (1962) 224.

27) Farmsworth, H., and Winch, R.P., "Photoelectric Work Functions of (100) and (111) Faces of Silver Single Crystals and Their Contact Potential Difference" , Phy. Rev., 58 (1940) 812.

28) Holm, R., "Electrical Contacts Handbook", Springer-Verlag, Berlin, Germany (1958).

29) Greenwood, J.A., "Constriction Resistance and the Real Area of Contact", Burndy Research Division, Report No. 38, Burndy Corp. , Norwalk, Conn. (May, 1966).

30) Aldrich, R.G., "A Sessile Drop Study of Liquid-Solid Adhesion in the Indium-Aluminum System Using Ultra-High Vacuum Techniques", Doctorate Dissertation, Syracuse University (July, 1966).

31) Keller, D.V., Jr., "Adhesion Between Atomically Pure Metallic Surfaces", Semi-Annual Report, NASA Grant NsG-483-1 (July, 1965). 